TRANSACTIONS OF THE

AMERICAN MATHEMATICAL SOCIETY

Volume 355, Number 3, Pages 865-899

S 0002-9947(02)03147-1

Article electronically published on November 1, 2002

\title{
ASYMPTOTICS FOR THE NONLINEAR DISSIPATIVE WAVE EQUATION
}

\author{
TOKIO MATSUYAMA
}

Dedicated to Professor Kunihiko Kajitani on the occasion of his sixtieth birthday

\begin{abstract}
We are interested in the asymptotic behaviour of global classical solutions to the initial-boundary value problem for the nonlinear dissipative wave equation in the whole space or the exterior domain outside a star-shaped obstacle. We shall treat the nonlinear dissipative term like $a_{1}(1+|x|)^{-\delta}\left|u_{t}\right|^{\beta} u_{t}$ $\left(a_{1}, \beta, \delta>0\right)$ and prove that the energy does not in general decay. Further, we can deduce that the classical solution is asymptotically free and the local energy decays at a certain rate as the time goes to infinity.
\end{abstract}

\section{INTRODUCTION}

Let $\Omega=\mathbb{R}^{N}$ or $\Omega \subset \mathbb{R}^{N}(N \geq 1)$ be an unbounded domain with a compact and smooth boundary $\partial \Omega$ contained in the ball $B_{\rho_{0}}=\left\{x \in \mathbb{R}^{N} ;|x| \leq \rho_{0}\right\}$ (if $N=1$, we assume $\Omega=(-\infty, \infty)$ or $\left.\Omega=\left(\rho_{0}, \infty\right)\right)$. We consider the initial-boundary value problem in $(0, \infty) \times \Omega$ :

$$
\text { (P) } \begin{cases}u_{t t}-\Delta u+a(t, x)\left|u_{t}\right|^{\beta} u_{t}=0, & (t, x) \in(0, \infty) \times \Omega, \\ u(0, x)=u_{0}(x), \quad u_{t}(0, x)=u_{1}(x), & x \in \Omega, \\ u(t, x)=0, & (t, x) \in(0, \infty) \times \partial \Omega,\end{cases}
$$

where $a(t, x)$ is a nonnegative function in $[0, \infty) \times \bar{\Omega}$ and $\beta>0$. When $\Omega$ is the whole space $\mathbb{R}^{N}$, we can drop the boundary condition. In this paper we are concerned with asymptotic behaviour such as energy nondecay and local energy decay for the $C^{2}$ solutions to the problem $(\mathrm{P})$.

Before we state assumptions, we shall introduce some function spaces and notation. Let $W^{k, p}(\Omega)(k=0,1,2, \ldots ; p \geq 1)$ be the usual Sobolev space with norm

$$
\|f\|_{W^{k, p}(\Omega)}=\left\{\sum_{|\alpha| \leq k} \int_{\Omega}\left|D_{x}^{\alpha} f(x)\right|^{p} d x\right\}^{1 / p}
$$

Received by the editors November 6, 2001 and, in revised form, July 4, 2002.

2000 Mathematics Subject Classification. Primary 35L05; Secondary 35L10.

Supported in part by a Grant-in-Aid for Scientific Research (C)(2)(No.11640213), Japan Society for the Promotion of Science.

The author would like to express his sincere gratitude to Professors K. Mochizuki, M. Nakao and M. Yamaguchi for several useful comments. He is also indebted to Professors Y. Shibata, N. Hayashi and T. Kobayashi, who pointed out the uniform decay estimate to him. The author thanks Doctor H. Nakazawa for advising him of the existence of scattering states. The author also thanks the referee for a careful reading of the manuscript.

(C)2002 American Mathematical Society 
where

$$
D_{x}^{\alpha}=\left(\frac{\partial}{\partial x_{1}}\right)^{\alpha_{1}}\left(\frac{\partial}{\partial x_{2}}\right)^{\alpha_{2}} \cdots\left(\frac{\partial}{\partial x_{N}}\right)^{\alpha_{N}} \quad\left(|\alpha|=\alpha_{1}+\alpha_{2}+\cdots+\alpha_{N}\right) .
$$

We write $H^{k}(\Omega)=W^{k, 2}(\Omega), H^{0}(\Omega)=L^{2}(\Omega)$ and $\|f\|_{L^{2}(\Omega)}=\|f\| . H_{0}^{1}(\Omega)$ and $H_{\nabla}(\Omega)$ are the completion of $C_{0}^{\infty}(\Omega)$ (the space of all test functions on $\Omega$ ) in the norms $\|\cdot\|_{H^{1}(\Omega)}$ and $\|\nabla \cdot\|$, respectively, where $\nabla=\left(\partial / \partial x_{1}, \partial / \partial x_{2}, \ldots, \partial / \partial x_{N}\right)$. $\mathcal{B}^{k}([0, \infty) \times \bar{\Omega})$ is the space of all functions whose derivatives up to the $k$-th order are all bounded and continuous on $[0, \infty) \times \bar{\Omega}$. The differential operators often used in this paper are as follows:

$$
\partial^{k}=\sum_{|\alpha|=k}\left(\frac{\partial}{\partial x_{1}}\right)^{\alpha_{1}}\left(\frac{\partial}{\partial x_{2}}\right)^{\alpha_{2}} \cdots\left(\frac{\partial}{\partial x_{N}}\right)^{\alpha_{N}}, \quad D_{t}^{j}=\frac{\partial^{j}}{\partial t^{j}} \quad(j=1,2, \cdots, N),
$$

and we define the (higher-order) energy of $u(t, x)$ by

$$
\begin{aligned}
& E\left(D_{t}^{j} u(t)\right)=\frac{1}{2}\left\{\left\|D_{t}^{j+1} u(t)\right\|^{2}+\left\|\nabla D_{t}^{j} u(t)\right\|^{2}\right\} \quad(j=0,1,2,3), \\
& I_{j}^{2}=E\left(D_{t}^{j} u(0)\right)=\frac{1}{2}\left\{\left\|D_{t}^{j+1} u(0)\right\|^{2}+\left\|\nabla D_{t}^{j} u(0)\right\|^{2}\right\} \quad(j=0,1,2,3) .
\end{aligned}
$$

We make the following hypotheses.

Hypothesis A. $a(t, x)$ is a nonnegative function in $[0, \infty) \times \bar{\Omega}$ and belongs to $C^{1}([0, \infty) \times \bar{\Omega})$, and is such that

$$
\left|a_{t}(t, x)\right|+|\nabla a(t, x)| \leq C a(t, x)
$$

for some $C>0$.

Hypothesis B. $a(t, x)$ satisfies

$$
0 \leq a(t, x) \leq a_{1}(1+|x|)^{-\delta} \quad \text { in }[0, \infty) \times \bar{\Omega}
$$

for some $a_{1}>0$ and $\delta \geq 0$.

Hypothesis C. $\mathbb{R}^{N} \backslash \Omega$ is star-shaped with respect to the origin if $\Omega \neq \mathbb{R}^{n}$.

Now, assume that

$$
\left\{u_{0}, u_{1}\right\} \in\left[H^{2}(\Omega) \cap H_{0}^{1}(\Omega)\right] \times\left[H_{0}^{1}(\Omega) \cap L^{2(\beta+1)}(\Omega)\right] .
$$

Then it is well known (cf. Lions and Strauss [5]) that if we assume Hypothesis A, then the problem $(\mathrm{P})$ has a unique global solution with the following properties:

(i) $u \in C\left([0, \infty) ; H_{0}^{1}(\Omega)\right)$, and $u$ satisfies the energy identity

$$
E(u(t))+\int_{s}^{t} \int_{\Omega} a\left|u_{t}\right|^{\beta+2} d x d \tau=E(u(s)) \quad \text { for } 0 \leq s<t .
$$

(ii) $u \in C\left([0, T] ; L^{2}(\Omega)\right)$ for any $T>0$.

(iii) $u_{t t}, \nabla u_{t}, \Delta u, a\left|u_{t}\right|^{\beta} u_{t} \in L^{\infty}\left(0, T ; L^{2}(\Omega)\right)$ for any $T>0$.

We see from the energy identity (1.1) that the energy decreases in $t>0$. Thus a question naturally arises whether it decays or not. The main purpose of this paper is to investigate the asymptotic behaviour of solutions for the "exterior problem". 
When $\Omega$ is the whole space $\mathbb{R}^{N}$, Mochizuki and Motai proved in [1] that if $a(t, x) \geq a_{0}(1+t+|x|)^{-\delta}(0 \leq \delta<1)$ and $0<\beta \leq 2(1-\delta) / N$, then the energy of the solution with the logarithmically weighted data decays like $\{\log (e+t)\}^{-\mu}(0<$ $\mu<2 / \beta)$, and if $0 \leq a(t, x) \leq a_{1}(1+|x|)^{-\delta}(0 \leq \delta \leq 1)$ and $\beta>2(1-\delta) /(N-1)$ $(N \geq 2)$, then the energy does not in general decay (see also Mochizuki [9], where the proof is not given in detail) and the solution is asymptotically free (see Motai and Mochizuki 14]). The decay estimate in [11 can be proved analogously in any exterior domain with a compact boundary. On the other hand, it seems difficult to discuss the energy nondecay problem in exterior domains without any assumption on the shape. For the linear dissipative case in an exterior domain outside a starshaped obstacle, we should refer to the work of Mochizuki and Nakazawa [12] (see also the work [6] of the present author in general exterior domains where the dissipation is effective around the boundary).

The first object in this paper is to discuss the energy nondecay of the solution to the problem $(\mathrm{P})$. In other words, we shall construct some special initial data for which the energy of the corresponding solution remains positive as $t$ goes to infinity. If $\mathbb{R}^{N} \backslash \Omega$ is star-shaped and $a(t, x)=O\left(|x|^{-\delta}\right)(|x| \rightarrow \infty)$ for some $\delta \geq 0$, we can prove the energy nondecay for some restrictive condition on $\beta$ (Theorem 2.1). We can also prove that if $\Omega$ is the whole space $\mathbb{R}^{3}$ and $a(t, x)$ satisfies $a(t, x)=$ $O\left(|x|^{-\delta} t^{-\eta}\right)$ for some $\delta>1$ and $\eta>0(t+|x| \rightarrow \infty)$, then the local energy decays at a certain rate as $t \rightarrow \infty$ (Theorem 2.4), which is the second object in this paper. In this argument, the boundedness or decay of $\left\|u_{t}(t)\right\|_{L^{\infty}}$ for the global $C^{2}$ solution $u$ will play an important role, and we can also claim that the scattering state exists in the exterior problem (Theorem 2.3), which is a sharper result than Theorem 2.1. Here, we should mention the result in [9] on the local energy decay, which showed that if $(N-1) \beta>2(1-\delta)$ and $\beta \geq-1$, then the local energy decays to 0 , but its rate was not determined.

For the present discussion we need the existence theorem for classical solutions to the problem $(\mathrm{P})$. This existence theorem was treated by many authors (see Nakao [15] and Sather [17] in a bounded domain, Shatah [18] in the whole space, and Hayashi [2] in the exterior domain outside the ball, Shibata and Tsutsumi [20] in exterior nontrapping domains, etc.). In all of these it was required for the existence of globally in time small-amplitude solutions that the initial data belongs to the "higher-order" Sobolev spaces. Our result concerning the existence theorem of the global $C^{2}$ solution $u$ in Theorem 2.2 is even new in the sense that the smallamplitude solutions belong to a weaker class, $H^{4} \times H^{3}$, than the previous ones. Since the energy does not in general decay, we cannot expect to obtain the decay estimate, and hence it is difficult to prove the global existence of classical solutions by the usual energy method.

In the case when $\Omega=\mathbb{R}^{3}$ or an exterior domain outside a star-shaped obstacle in $\mathbb{R}^{3}$, if $a(t, x)=O\left(|x|^{-\delta}\right)(|x| \rightarrow \infty)$ for some $\delta>1$, we shall use the weighted energy method to obtain the space-time integrability of $a\left|D_{t}^{j} u\right|^{2}(j=1,2,3,4)$ in Proposition 5.5, and as a result, we can deduce that the wave operator $\partial_{t}^{2}-\Delta$ has a dissipative effect like $a_{1}(1+|x|)^{-\delta} u_{t}$ for some $a_{1}>0$ and $\delta>1$. From this observation it follows that a nonlinear term like $a u_{t}^{3}$ can be absorbed into the space-time estimates of $a\left|D_{t}^{j} u\right|^{2}$, which enables us to assure the existence of global classical solutions in Theorem 2.2. 


\section{Statement of Results}

We now consider the initial-boundary value problem for the free wave equation with the same initial data as the problem $(\mathrm{P})$ :

$$
(\mathrm{P})_{0} \quad \begin{cases}w_{t t}-\Delta w=0, & (t, x) \in(0, \infty) \times \Omega, \\ w(0, x)=u_{0}(x), \quad w_{t}(0, x)=u_{1}(x), & x \in \Omega, \\ w(t, x)=0, & (t, x) \in(0, \infty) \times \partial \Omega .\end{cases}
$$

It is well known (cf. Ikawa [3]) that if $\left\{u_{0}, u_{1}\right\}$ belongs to $H^{k+1}(\Omega) \times H^{k}(\Omega), k$ being an integer with $k \geq[N / 2]+1([N / 2]=$ the integer part of $N / 2)$, and satisfies the compatibility condition of order $k$, that is, $w_{j} \in H_{0}^{1}(\Omega)\left(j=0,1, \ldots, k ; w_{0}=u_{0}\right.$, $\left.w_{1}=u_{1}\right)$ and $w_{k+1} \in L^{2}(\Omega)$, where $\left\{w_{j}\right\}$ are defined inductively by

$$
w_{j}=\Delta w_{j-2}(j=2,3, \cdots, k+1),
$$

then the finite energy solution $w(t, x)$ belongs, in fact, to

$$
X^{k}(0, \infty) \equiv \bigcap_{j=0}^{k} C^{j}\left([0, \infty) ; H^{k+1-j}(\Omega) \cap H_{0}^{1}(\Omega)\right) \cap C^{k+1}\left([0, \infty) ; L^{2}(\Omega)\right)
$$

and satisfies

$$
\sup _{t \geq 0}\left\|w_{t}(t)\right\|_{L^{\infty}} \leq C_{k} \equiv C\left(\|w(0)\|_{H^{k+1}},\left\|w_{t}(0)\right\|_{H^{k}}\right) .
$$

In order to state the result, we need

$$
\int_{0}^{\infty} \int_{\Omega} a\left|w_{t}(t)\right|^{\beta+2} d x d t \leq C_{\beta} E(w(0))
$$

for some $C_{\beta} \equiv C\left(\beta,\|w(0)\|_{H^{k+1}},\left\|w_{t}(0)\right\|_{H^{k}}\right)>0(k \geq[N / 2]+1)$ independent of $t$, which will be shown in Lemma 4.2 below. Our first result reads as follows.

Theorem 2.1. Suppose Hypotheses A, B, C hold, and $\left\{u_{0}, u_{1}\right\} \neq\{0,0\}$.

(i) Let $N \neq 2, \delta>1$ and $\beta>0$. Assume further that $\left\{u_{0}, u_{1}\right\}$ belongs to $H^{[N / 2]+2}(\Omega) \times H^{[N / 2]+1}(\Omega)$ and satisfies the compatibility condition of order $[N / 2]+$ 1 in the sense of (2.1). If we take $\sigma \equiv \sigma\left(u_{0}, u_{1}\right)>0$ so that

$$
\int_{\sigma}^{\infty} \int_{\Omega} a\left|w_{t}(t)\right|^{\beta+2} d x d t<2^{\beta+2} E(u(0)),
$$

then the energy $E\left(u^{(\sigma)}(t)\right)$ of the solution $u^{(\sigma)}(t)$ in the class $X^{1}(0, \infty)$ to the problem $(\mathrm{P})$ with the initial data $\left\{u_{0}, u_{1}\right\}$ replaced by $\left\{w(\sigma), w_{t}(\sigma)\right\}$ never decays to 0 as $t \rightarrow \infty$.

(ii) Let $N \geq 3,0 \leq \delta \leq 1$ and

$$
\beta>\frac{2}{N-1}-\frac{2 \delta}{N} .
$$

Suppose that $\mathbb{R}^{N} \backslash \Omega$ is convex if $\Omega \neq \mathbb{R}^{N}$. Assume that $\left\{u_{0}, u_{1}\right\}$ belongs to

$$
\left[H^{2[N / 2]+3}(\Omega) \cap W^{2[N / 2]+4,1}(\Omega)\right] \times\left[H^{2[N / 2]+2}(\Omega) \cap W^{2[N / 2]+3,1}(\Omega)\right]
$$

and satisfies the compatibility condition of order $2[N / 2]+2$ in the sense of (2.1). Then the assertion in (i) remains valid. In particular, if $\Omega$ is the whole space $\mathbb{R}^{N}$ and the initial data $\left\{u_{0}, u_{1}\right\}$ belongs to

$$
\left[H^{[N / 2]+2}\left(\mathbb{R}^{N}\right) \cap W^{[N / 2]+3,1}\left(\mathbb{R}^{N}\right)\right] \times\left[H^{[N / 2]+1}\left(\mathbb{R}^{N}\right) \cap W^{[N / 2]+2,1}\left(\mathbb{R}^{N}\right)\right],
$$


then the assertion in (i) remains valid even if $N \geq 2$.

Remark. When $\Omega=\mathbb{R}^{N}(N \geq 2)$, Mochizuki and Motai assumed in [11] that $\left\{u_{0}, u_{1}\right\}, \beta$ and $\delta$ should satisfy

$$
\begin{gathered}
\sum_{|\alpha|,|\gamma| \leq[N / 2]+2}\left\|x^{\alpha} \nabla^{\gamma} u_{0}\right\|+\sum_{|\alpha|,|\gamma| \leq[N / 2]+1}\left\|x^{\alpha} \nabla^{\gamma} u_{1}\right\|<\infty, \\
\beta>\frac{2}{N}-\frac{2 \delta}{N} \quad \text { and } \quad 0 \leq \delta \leq 1
\end{gathered}
$$

to obtain that $E\left(u^{(\sigma)}(t)\right)$ remains positive as $t$ goes to infinity. Thus part (ii) in Theorem 2.1 imposes an $L^{1}$ condition and removes the weight condition on (2.3).

In order to discuss the existence of a scattering state and the local energy decay, we need the existence of the globally small-amplitude $C^{2}$ solution to the problem $(\mathrm{P})$. For this, we must restrict the condition on the power nonlinearity of the dissipation to $a u_{t}^{2 m+1}(\beta=2 m)$.

Let $\Omega$ be the whole space $\mathbb{R}^{3}$ or the exterior domain in $\mathbb{R}^{3}$ with a compact boundary. Assume $m=1,2$ or $m \geq 3$, which assures that the nonlinear dissipative term is of class $C^{3}$. Then we consider the initial-boundary value problem

$$
(\mathrm{P})_{m} \begin{cases}u_{t t}-\Delta u+a(t, x) u_{t}^{2 m+1}=0, & (t, x) \in(0, \infty) \times \Omega, \\ u(0, x)=u_{0}(x), \quad u_{t}(0, x)=u_{1}(x), & x \in \Omega, \\ u(t, x)=0, & (t, x) \in(0, \infty) \times \partial \Omega .\end{cases}
$$

We assume that $\left\{u_{0}, u_{1}\right\} \in H^{4}(\Omega) \times H^{3}(\Omega)$ satisfies the compatibility condition of order 3 :

$$
D_{t}^{j} u(0, \cdot) \in H_{0}^{1}(\Omega) \quad(j=0,1,2,3) \quad \text { and } \quad D_{t}^{4} u(0, \cdot) \in L^{2}(\Omega) .
$$

Furthermore, we impose the other assumption as

Hypothesis D. $a(t, x)$ belongs to $C^{3}([0, \infty) \times \bar{\Omega})$ and satisfies

$$
\sum_{j+|\alpha| \leq 3}\left|D_{t}^{j} D_{x}^{\alpha} a(t, x)\right| \leq C a(t, x) \quad \text { in }[0, \infty) \times \bar{\Omega}
$$

for some $C>0$.

Then the existence theorem for $C^{2}$ solutions reads as follows.

Theorem 2.2. Let $N=3$ and $\delta>1$. Suppose Hypothesis D holds. Then we have the following assertions.

(i) Assume Hypotheses B, C and $m=1,2$ or $m \geq 3$. Then, there exist positive constants $\varepsilon_{0}, C_{0}$ and $C_{1}$ such that if the initial data $\left\{u_{0}, u_{1}\right\} \in H^{4}(\Omega) \times H^{3}(\Omega)$ satisfies

$$
\begin{gathered}
\left\|\nabla u_{0}\right\|_{H^{3}}+\sum_{j=0}^{3}\left\|D_{t}^{j+1} u(0)\right\|_{H^{3-j}}<C_{0} \varepsilon_{0}, \\
\left\|u_{t}(0)\right\|_{L^{\infty}}+\left\|u_{t t}(0)\right\|_{L^{\infty}}+\left\|\nabla u_{t}(0)\right\|_{L^{\infty}}<\varepsilon_{0},
\end{gathered}
$$


and the compatibility condition of order 3 in the sense of (2.5), then the problem $(\mathrm{P})_{m}$ admits a unique solution $u \in C^{2}([0, \infty) \times \bar{\Omega})$ so that

$$
\begin{aligned}
& \sup _{t \geq 0}\left\{\|\nabla u(t)\|_{H^{3}}+\sum_{j=0}^{3}\left\|D_{t}^{j+1} u(t)\right\|_{H^{3-j}}\right\} \leq C_{0} C_{1} \varepsilon_{0}, \\
& \sup _{t \geq 0}\left\{\left\|u_{t}(t)\right\|_{L^{\infty}}+\left\|u_{t t}(t)\right\|_{L^{\infty}}+\left\|\nabla u_{t}(t)\right\|_{L^{\infty}}\right\} \leq \varepsilon_{0} .
\end{aligned}
$$

(ii) Let $\Omega=\mathbb{R}^{3}$, and let $m$ be a number with $m=2$ or $m \geq 3$. Suppose that the data $u_{0}, u_{1}$ satisfies $\left\{u_{0}, u_{1}\right\} \in\left[H^{4}\left(\mathbb{R}^{3}\right) \cap W^{4,1}\left(\mathbb{R}^{3}\right)\right] \times\left[H^{3}\left(\mathbb{R}^{3}\right) \cap W^{3,1}\left(\mathbb{R}^{3}\right)\right]$, (2.6) and (2.7). Assume further that $a(t, x)$ satisfies

$$
0 \leq a(t, x) \leq a_{1}(1+t)^{-\eta}(1+|x|)^{-\delta}
$$

for some $a_{1}>0, \eta>0$ and $\delta>1$. If we choose $\varepsilon_{0}$ small enough, then the $C^{2}$ solution $u$ satisfies

$$
\begin{aligned}
\sup _{t \geq 0}\{ & \left.(1+t)\left(\left\|u_{t}(t)\right\|_{L^{\infty}}+\|\nabla u(t)\|_{L^{\infty}}\right)\right\} \\
& \leq C\left(\varepsilon_{0}\right)\left(\left\|u_{0}\right\|_{W^{4,1}}+\left\|u_{0}\right\|_{H^{3}}+\left\|u_{1}\right\|_{W^{3,1}}+\left\|u_{1}\right\|_{H^{2}}\right) .
\end{aligned}
$$

Based on the $L^{\infty}$ estimate (2.9), we can argue the existence of a scattering state for our problem, which reveals the meaning of the energy nondecay in Theorem 2.1 (i). That is, we have the following.

Theorem 2.3. For the solution $u(t, x)$ in the sense of Theorem 2.2 (i) to the problem $(\mathrm{P})_{m}$ with the data $\left\{u_{0}, u_{1}\right\}$, there exists a pair $\left\{u_{0}^{+}, u_{1}^{+}\right\} \in E$ such that

$$
\left\|u(t)-w^{+}(t)\right\|_{E} \rightarrow 0 \quad \text { as } t \rightarrow \infty
$$

where $E$ represents the energy space $H_{\nabla}(\Omega) \times L^{2}(\Omega)$ and $w^{+}(t)$ is the solution to the problem $(\mathrm{P})_{0}$ with $\left\{u_{0}, u_{1}\right\}$ replaced by $\left\{u_{0}^{+}, u_{1}^{+}\right\}$.

Remark. When $\Omega=\mathbb{R}^{N}(N \geq 2)$, Motai and Mochizuki 14 showed that if $\beta>$ $2 /(N-1)$ and $\delta=0$, then the scattering state exists. Therefore, Theorem 2.3 is new in the sense of the exterior problem.

Finally, let us state the result on the local energy decay.

Theorem 2.4. Let $\Omega$ be the whole space $\mathbb{R}^{3}$ and $u(t, x)$ the solution in the sense of Theorem 2.2 (ii) with $\operatorname{supp} u_{0} \cup \operatorname{supp} u_{1} \subset B_{R}$ for any fixed number $R>0$. Then

$$
\frac{1}{2} \int_{B_{R}}\left\{\left|u_{t}(t, x)\right|^{2}+|\nabla u(t, x)|^{2}\right\} d x \leq C\left(R, \varepsilon_{0}\right)(1+t)^{-\min \{2(2 m+\eta), 2(\delta-1)\}}
$$

for all $t \geq 0$.

Remark. When $a(t, x)$ has compact support, $E_{l o c, R}(t)$ decays exponentially to 0 as $t$ goes to infinity. For the details, see [7]. 


\section{Preliminaries}

We state the following well-known regularity theorem of elliptic equations.

Lemma 3.1 (1], Gilbarg and Trudinger, p. 187, Theorem 8.13). Let $m$ be a nonnegative integer. If $\Delta u \in H^{m}(\Omega)$ and $u \in H_{0}^{1}(\Omega)$, then $u \in H^{m+2}(\Omega)$ and

$$
\|u\|_{H^{m+2}} \leq C_{m}\left(\|\Delta u\|_{H^{m}}+\|u\|\right)
$$

for some positive constant $C_{m}$ depending on $m$.

Lemma 3.2 ([4], Ladyzhenskaya). Let $H_{\nabla}^{2}(\Omega)=\left\{u \in H_{\nabla}(\Omega) ; \Delta u \in L^{2}(\Omega)\right\}$. If $u \in H_{\nabla}^{2}(\Omega)$, then $D_{x}^{\alpha} u \in L^{2}(\Omega)(|\alpha|=2)$ and there exists a constant $C>0$ such that

$$
\sum_{|\alpha|=2}\left\|D_{x}^{\alpha} u\right\| \leq C(\|\nabla u\|+\|\Delta u\|)
$$

To obtain a priori estimates of higher-order derivatives, we need $L^{1}$ bounds for the weighted energy. For this, let us introduce the positive smooth function $\varphi(r)$ of $r=|x|$ such that

$$
\varphi(r) \text { is bounded, monotone increasing and satisfies } \varphi(r) \geq r \varphi^{\prime}(r) .
$$

Lemma 3.3. Let $u(t, x)$ be the solution to the problem $(\mathrm{P})$. Then we have

$$
\frac{1}{2} \frac{\partial}{\partial t}\left\{u_{t}^{2}+|\boldsymbol{\theta}|^{2}+\frac{(N-1)(N-3)}{4 r^{2}} u^{2}\right\}-\nabla \cdot\left(\boldsymbol{\theta} u_{t}\right)+a\left|u_{t}\right|^{\beta+2}=0
$$

and

$$
\begin{aligned}
& \frac{\partial}{\partial t}\{\varphi(r)\left.(\widetilde{x} \cdot \boldsymbol{\theta}) u_{t}\right\} \\
&-\nabla \cdot\left[\varphi(r)(\widetilde{x} \cdot \boldsymbol{\theta}) \boldsymbol{\theta}+\frac{1}{2} \varphi(r) \widetilde{x}\left\{u_{t}^{2}-|\boldsymbol{\theta}|^{2}-\frac{(N-1)(N-3)}{4 r^{2}} u^{2}\right\}\right] \\
&+\frac{1}{2} \varphi^{\prime}(r) u_{t}^{2}+\left\{\frac{1}{r} \varphi(r)-\frac{1}{2} \varphi^{\prime}(r)\right\}|\boldsymbol{\theta}|^{2}-\left\{\frac{1}{r} \varphi(r)-\varphi^{\prime}(r)\right\}(\widetilde{x} \cdot \boldsymbol{\theta})^{2} \\
& \quad+a \varphi(r)(\widetilde{x} \cdot \boldsymbol{\theta})\left|u_{t}\right|^{\beta} u_{t}+\left\{\frac{1}{r} \varphi(r)-\frac{1}{2} \varphi^{\prime}(r)\right\} \frac{(N-1)(N-3)}{4 r^{2}} u^{2}=0,
\end{aligned}
$$

where we set

$$
\boldsymbol{\theta}=\nabla u+\frac{N-1}{2 r} \widetilde{x} u \quad\left(\widetilde{x}=\frac{x}{r}\right)
$$

Proof. The proof can be done along the same lines as in the book of Mochizuki [10]. For the convenience of the readers we give it completely.

Setting $v(t, x)=r^{(N-1) / 2} u(t, x)$, we easily see that $\boldsymbol{\theta}=r^{-(N-1) / 2} \nabla v$, and the equation can be rewritten as

$$
v_{t t}-\Delta v+\frac{N-1}{r} \widetilde{x} \cdot \nabla v+a r^{-\beta(N-1) / 2}\left|v_{t}\right|^{\beta} v_{t}+\frac{(N-1)(N-3)}{4 r^{2}} v=0 .
$$

In the calculations below, we shall construct the divergence form. Multiplying the equation (3.4) by $r^{1-N} v_{t}$, we have

$$
\begin{gathered}
\left\{v_{t t}+a r^{-\beta(N-1) / 2}\left|v_{t}\right|^{\beta} v_{t}+\frac{(N-1)(N-3)}{4 r^{2}} v\right\} r^{1-N} v_{t} \\
=\frac{\partial}{\partial t}\left\{\frac{1}{2} u_{t}^{2}+\frac{(N-1)(N-3)}{8 r^{2}} u^{2}\right\}+a\left|u_{t}\right|^{\beta+2}
\end{gathered}
$$


and

$$
\begin{aligned}
\left(-\Delta v+\frac{N-1}{r} \widetilde{x} \cdot \nabla v\right) r^{1-N} v_{t}= & -\nabla \cdot\left\{r^{1-N}(\nabla v) v_{t}\right\}+r^{1-N} \nabla v \cdot \nabla v_{t} \\
& +\left\{\nabla\left(r^{1-N}\right) \cdot \nabla v\right\} v_{t}+\frac{N-1}{r}(\widetilde{x} \cdot \nabla v)\left(r^{1-N} v_{t}\right) \\
= & -\nabla \cdot\left(\boldsymbol{\theta} u_{t}\right)+\frac{1}{2} \frac{\partial}{\partial t}|\boldsymbol{\theta}|^{2},
\end{aligned}
$$

which imply the identity 3.2 .

Multiply the equation (3.4) by $r^{1-N} \varphi(r)(\widetilde{x} \cdot \nabla v)$. Then the time derivative terms become

$$
\begin{array}{r}
\left\{v_{t t}+a r^{-\beta(N-1) / 2}\left|v_{t}\right|^{\beta} v_{t}\right\} r^{1-N} \varphi(r)(\widetilde{x} \cdot \nabla v)=\frac{\partial}{\partial t}\left\{\varphi(r)(\widetilde{x} \cdot \boldsymbol{\theta}) u_{t}\right\}-\varphi(r)\left(\widetilde{x} \cdot \boldsymbol{\theta}_{t}\right) u_{t} \\
+a \varphi(r)(\widetilde{x} \cdot \boldsymbol{\theta})\left|u_{t}\right|^{\beta} u_{t}
\end{array}
$$

and the other terms become

$$
\begin{aligned}
& \left\{-\Delta v+\frac{N-1}{r} \widetilde{x} \cdot \nabla v+\frac{(N-1)(N-3)}{4 r^{2}} v\right\} r^{1-N} \varphi(r)(\widetilde{x} \cdot \nabla v) \\
= & -\Delta v \cdot r^{1-N} \varphi(r)(\widetilde{x} \cdot \nabla v)+\frac{N-1}{r} \varphi(r)(\widetilde{x} \cdot \boldsymbol{\theta})^{2} \\
& +\frac{(N-1)(N-3)}{4 r^{2}} \varphi(r)(\widetilde{x} \cdot \nabla u) u+\frac{N-1}{2 r} \cdot \frac{(N-1)(N-3)}{4 r^{2}} \varphi(r) u^{2} \\
= & -\Delta v \cdot r^{1-N} \varphi(r)(\widetilde{x} \cdot \nabla v)+\frac{N-1}{r} \varphi(r)(\widetilde{x} \cdot \theta)^{2} \\
& +\nabla \cdot\left\{\frac{(N-1)(N-3)}{8 r^{2}} \varphi(r) \widetilde{x} u^{2}\right\}+\left\{\frac{1}{r} \varphi(r)-\frac{1}{2} \varphi^{\prime}(r)\right\} \frac{(N-1)(N-3)}{4 r^{2}} u^{2},
\end{aligned}
$$

which imply

$$
\begin{aligned}
& \frac{\partial}{\partial t}\left\{\varphi(r)(\widetilde{x} \cdot \boldsymbol{\theta}) u_{t}\right\}-\varphi(r)\left(\widetilde{x} \cdot \boldsymbol{\theta}_{t}\right) u_{t}+a \varphi(r)(\widetilde{x} \cdot \boldsymbol{\theta})\left|u_{t}\right|^{\beta} u_{t} \\
& -\Delta v \cdot r^{1-N} \varphi(r)(\widetilde{x} \cdot \nabla v)+\frac{N-1}{r} \varphi(r)(\widetilde{x} \cdot \boldsymbol{\theta})^{2} \\
& +\nabla \cdot\left\{\frac{(N-1)(N-3)}{8 r^{2}} \varphi(r) \widetilde{x} u^{2}\right\}+\left\{\frac{1}{r} \varphi(r)-\frac{1}{2} \varphi^{\prime}(r)\right\} \frac{(N-1)(N-3)}{4 r^{2}} u^{2}=0 .
\end{aligned}
$$

By simple calculations, the second term in the left-hand side of the identity (3.5) becomes

$$
\begin{aligned}
-\varphi(r)\left(\widetilde{x} \cdot \boldsymbol{\theta}_{t}\right) u_{t} & =-\varphi(r)\left(\widetilde{x} \cdot \nabla u_{t}\right) u_{t}-\frac{N-1}{2 r} \varphi(r) u_{t}^{2} \\
& =-\frac{1}{2} \nabla \cdot\left\{\varphi(r) \widetilde{x} u_{t}^{2}\right\}+\frac{1}{2} \varphi^{\prime}(r) u_{t}^{2}
\end{aligned}
$$


and the fourth term in the left-hand side of the identity (3.5) becomes

$$
\begin{aligned}
& -\Delta v \cdot r^{1-N} \varphi(r)(\widetilde{x} \cdot \nabla v) \\
=-\nabla \cdot\{\varphi(r)(\widetilde{x} \cdot \boldsymbol{\theta}) \boldsymbol{\theta}\} & +r^{1-N}(\nabla \varphi \cdot \nabla v)(\widetilde{x} \cdot \nabla v)+\varphi(r)\left\{\nabla\left(r^{1-N}\right) \cdot \nabla v\right\}(\widetilde{x} \cdot \nabla v) \\
=-\nabla \cdot\{\varphi(r)(\widetilde{x} \cdot \boldsymbol{\theta}) \boldsymbol{\theta}\} & +\varphi^{\prime}(r)(\widetilde{x} \cdot \boldsymbol{\theta})^{2}+(1-N) r^{-N} \varphi(r)(\widetilde{x} \cdot \nabla v)^{2} \\
& +\varphi(r) r^{1-N} \nabla v \cdot \nabla(\widetilde{x} \cdot \nabla v)
\end{aligned}
$$

Here, we note that the last term in the right-hand side of (3.7) continually becomes

$$
\begin{aligned}
\varphi(r) & r^{1-N} \nabla v \cdot \nabla(\widetilde{x} \cdot \nabla v) \\
= & \frac{1}{r} \varphi(r)\left\{|\boldsymbol{\theta}|^{2}-(\widetilde{x} \cdot \boldsymbol{\theta})^{2}\right\}+\frac{1}{2} \varphi(r) r^{1-N} \widetilde{x} \cdot \nabla\left(|\nabla v|^{2}\right) \\
= & \frac{1}{r} \varphi(r)\left\{|\boldsymbol{\theta}|^{2}-(\widetilde{x} \cdot \boldsymbol{\theta})^{2}\right\}+\frac{1}{2} \varphi(r) r^{1-N} \widetilde{x} \cdot \nabla\left(r^{1-N}|\nabla v|^{2} \cdot r^{N-1}\right) \\
= & \frac{1}{r} \varphi(r)\left\{|\boldsymbol{\theta}|^{2}-(\widetilde{x} \cdot \boldsymbol{\theta})^{2}\right\}+\frac{1}{2} \varphi(r) \widetilde{x} \cdot \nabla\left(|\boldsymbol{\theta}|^{2}\right)+\frac{N-1}{2 r} \varphi(r)|\boldsymbol{\theta}|^{2} \\
& =\frac{1}{r} \varphi(r)\left\{|\boldsymbol{\theta}|^{2}-(\widetilde{x} \cdot \boldsymbol{\theta})^{2}\right\}+\nabla \cdot\left\{\frac{1}{2} \varphi(r) \widetilde{x}|\boldsymbol{\theta}|^{2}\right\}-\frac{1}{2} \varphi^{\prime}(r)|\boldsymbol{\theta}|^{2} .
\end{aligned}
$$

Hence, it follows from (3.7) and (3.8) that

$$
\begin{aligned}
& \left.\left.-\Delta v \cdot r^{1-N} \varphi(r)(\widetilde{x} \cdot \nabla v)\right\}\right) \\
= & -\nabla \cdot\left\{\varphi(r)(\widetilde{x} \cdot \boldsymbol{\theta}) \boldsymbol{\theta}-\frac{1}{2} \varphi(r) \widetilde{x}|\boldsymbol{\theta}|^{2}\right\}-\varphi(r) \cdot \frac{N-1}{r}(\widetilde{x} \cdot \boldsymbol{\theta})^{2} \\
& +\left\{\frac{1}{r} \varphi(r)-\frac{1}{2} \varphi^{\prime}(r)\right\}|\boldsymbol{\theta}|^{2}-\left\{\frac{1}{r} \varphi(r)-\varphi^{\prime}(r)\right\}(\widetilde{x} \cdot \boldsymbol{\theta})^{2} .
\end{aligned}
$$

Combining (3.5), (3.6) and (3.9), we get the identity (3.3).

\section{THE ENERGY NONDECAY}

In this section we will observe that the energy does not in general decay under the assumptions in Theorem 2.1. The following identity is our starting point.

Lemma 4.1. Assume that $\left\{u_{0}, u_{1}\right\} \in\left[H^{2}(\Omega) \cap H_{0}^{1}(\Omega)\right] \times H_{0}^{1}(\Omega)$. Let $w(t, x)$ be the $X^{1}(0, \infty)$-solution to the problem $(\mathrm{P})_{0}$, and let $u^{(\sigma)}(t, x)$ be the $X^{1}(0, \infty)$-solution to the problem $(\mathrm{P})$ with the data $\left\{u_{0}, u_{1}\right\}$ replaced by $\left\{w(\sigma), w_{t}(\sigma)\right\}$ for any fixed $\sigma>0$. Then we have

$$
\left(u^{(\sigma)}(t), w(t+\sigma)\right)_{E}+\frac{1}{2} \int_{0}^{t} \int_{\Omega} a\left|u_{t}^{(\sigma)}(\tau)\right|^{\beta} u_{t}^{(\sigma)}(\tau) w_{t}(\tau+\sigma) d x d \tau=E(u(0)),
$$

where $(\cdot, \cdot)_{E}$ is the inner product in the energy space

$$
(u(t), w(t))_{E}=\frac{1}{2} \int_{\Omega}\left(u_{t} w_{t}+\nabla u \cdot \nabla w\right) d x .
$$

Proof. Multiply the equation in (P) by $u_{t}^{(\sigma)}$ and integrate by parts over $(\sigma, t) \times \Omega$ $(0<\sigma<t)$. Then, noting that $\left(u^{(\sigma)}(0), w(\sigma)\right)_{E}=E(u(0))$, we get (4.1). 
When $\delta>1$, the following integrability of $a\left|w_{t}\right|^{\beta+2}$ will play a crucial role in constructing a special data for which the energy never decays as $t \rightarrow \infty$.

Lemma 4.2. Let $N \neq 2$. Assume Hypotheses $\mathrm{B}$ and $\mathrm{C}$, and let $\delta>1$ and $\beta>0$. Let $w(t, x)$ be the solution in the class $X^{[N / 2]+1}(0, \infty)$ to the problem $(\mathrm{P})_{0}$ with the data $\left\{u_{0}, u_{1}\right\} \in H^{[N / 2]+2}(\Omega) \times H^{[N / 2]+1}(\Omega)$ satisfying the compatibility condition of order $[N / 2]+1$ in the sense of (2.1). Then there exists a constant $C_{\beta}>0$, depending on $\beta,\|w(0)\|_{H^{[N / 2]+2}}$ and $\left\|w_{t}(0)\right\|_{H^{[N / 2]+1}}$, such that

$$
\int_{0}^{\infty} \int_{\Omega} a\left|w_{t}\right|^{\beta+2} d x d t \leq C_{\beta} E(w(0))
$$

Proof. First we shall prove that

$$
\int_{0}^{\infty} \int_{\Omega} a w_{t}^{2} d x d t \leq C E(u(0))
$$

We apply Lemma 3.3 to $w$. Noting that $a \equiv 0$, we see that

$$
X_{t}+Z=\nabla \cdot Y
$$

where we set

$$
\left\{\begin{aligned}
X= & \frac{1}{2}\left\{w_{t}^{2}+|\boldsymbol{\theta}|^{2}+\frac{(N-1)(N-3)}{4 r^{2}} w^{2}\right\}+\varphi(r)(\widetilde{x} \cdot \boldsymbol{\theta}) w_{t} \\
\boldsymbol{Y}= & \boldsymbol{\theta} w_{t}+\varphi(r)\left\{(\widetilde{x} \cdot \boldsymbol{\theta}) \boldsymbol{\theta}+\frac{1}{2} \widetilde{x}\left[w_{t}^{2}-|\boldsymbol{\theta}|^{2}-\frac{(N-1)(N-3)}{4 r^{2}} w^{2}\right]\right\} \\
Z= & \frac{1}{2} \varphi^{\prime}(r)\left(w_{t}^{2}+|\boldsymbol{\theta}|^{2}\right)+\left\{\frac{1}{r} \varphi(r)-\varphi^{\prime}(r)\right\}\left\{|\boldsymbol{\theta}|^{2}-(\widetilde{x} \cdot \boldsymbol{\theta})^{2}\right\} \\
& +\left\{\frac{1}{r} \varphi(r)-\frac{1}{2} \varphi^{\prime}(r)\right\} \frac{(N-1)(N-3)}{4 r^{2}} w^{2} .
\end{aligned}\right.
$$

Then we integrate (4.4) over $(0, t) \times \Omega$ to obtain

$$
\left.\int_{\Omega} X(\tau, x) d x\right|_{\tau=0} ^{\tau=t}+\int_{0}^{t} \int_{\Omega} Z(\tau, x) d x d \tau=\int_{0}^{t} \int_{\partial \Omega} \boldsymbol{Y}(\tau, x) \cdot \boldsymbol{\nu}(x) d S d \tau
$$

where $\boldsymbol{\nu}(x)$ is the unit outward normal vector at $x \in \partial \Omega$. Of course, when $\Omega$ is the whole space $\mathbb{R}^{N}$, we can drop the boundary integral term in (4.5). Since $\varphi(r)$ is bounded, there exists a constant $C_{\varphi}>0$ such that

$$
\sup _{t \geq 0} \int_{\Omega}|X(t, x)| d x \leq C_{\varphi} E(w(0))
$$

where we have used $E(w(t))=E(w(0))$ and the Hardy inequality

$$
\int_{\Omega} \frac{u(x)^{2}}{r^{2}} d x \leq C\|\nabla u\|^{2} \quad \text { for } u \in H_{0}^{1}(\Omega) \text { and } N \geq 3 .
$$

Needless to say, when $N=1$, (4.6) is obvious. By means of the boundary condition $\left.w\right|_{\partial \Omega}=0$ and Hypothesis $\mathrm{C}$ we see that $w_{t}=0, \boldsymbol{\theta}=\nabla w=\left(\frac{\partial w}{\partial \boldsymbol{\nu}}\right) \boldsymbol{\nu}$ and $\widetilde{x} \cdot \boldsymbol{\nu}(x)<0$ on $\partial \Omega$. Hence we have

$$
\int_{0}^{t} \int_{\partial \Omega} \boldsymbol{Y}(\tau, x) \cdot \boldsymbol{\nu}(x) d S d \tau=\frac{1}{2} \int_{0}^{t} \int_{\partial \Omega} \varphi(r)\{\widetilde{x} \cdot \boldsymbol{\nu}(x)\}\left(\frac{\partial w}{\partial \boldsymbol{\nu}}\right)^{2} d S d \tau \leq 0 .
$$

Further, since we have $\left(r^{-1} \varphi-\varphi^{\prime}\right)\left\{|\boldsymbol{\theta}|^{2}-(\widetilde{x} \cdot \boldsymbol{\theta})^{2}\right\} \geq 0$ and $r^{-1} \varphi-2^{-1} \varphi^{\prime} \geq 2^{-1} \varphi^{\prime}$, it follows that

$$
Z \geq \frac{1}{2} \varphi^{\prime}(r)\left\{w_{t}^{2}+|\boldsymbol{\theta}|^{2}+\frac{(N-1)(N-3)}{4 r^{2}} w^{2}\right\}
$$


We put

$$
\varphi(r)=\gamma\left\{1-\frac{1}{\mu(1+r)^{\delta-1}}\right\},
$$

where $\delta>1, \gamma>0$ and $\mu>1$. Then

$$
\varphi^{\prime}(r)=\frac{\gamma(\delta-1)}{\mu}(1+r)^{-\delta},
$$

and it follows that $\varphi(r) \geq r \varphi^{\prime}(r)$. Thus (3.1) is satisfied for this $\varphi(r)$. Therefore, it follows from Hypothesis B and (4.5)-(4.9) that (4.3) is valid.

Thus the estimate (4.2) is an immediate consequence of the estimates (2.2) and (4.3), as follows:

$$
\int_{0}^{\infty} \int_{\Omega} a\left|w_{t}\right|^{\beta+2} d x d t \leq\left\{\sup _{t \geq 0}\left\|w_{t}(t)\right\|_{L^{\infty}}^{\beta}\right\} \int_{0}^{\infty} \int_{\Omega} a w_{t}^{2} d x d t \leq C_{\beta} E(u(0)) .
$$

This ends the proof of Lemma 4.2.

Now we shall prove Theorem 2.1 (i) by a contradiction argument. Let $w(t, x)$ be as in Lemma 4.2. By virtue of Lemma 4.2 we can take $\sigma \equiv \sigma\left(u_{0}, u_{1}\right)>0$ so that

$$
\int_{\sigma}^{\infty} \int_{\Omega} a\left|w_{t}(t)\right|^{\beta+2} d x d t<2^{\beta+2} E(u(0)) .
$$

For such a time $\sigma>0$ we consider the $X^{1}(0, \infty)$-solution $u^{(\sigma)}(t, x)$ to the problem (P) with the data $\left\{u_{0}, u_{1}\right\}$ replaced by $\left\{w(\sigma), w_{t}(\sigma)\right\}$. We suppose that the energy $E\left(u^{(\sigma)}(t)\right)$ of $u^{(\sigma)}(t, x)$ decays to 0 as $t \rightarrow \infty$ and argue to a contradiction. Letting $t \rightarrow \infty$, we see from Lemma 4.1 that

$$
\int_{0}^{\infty} \int_{\Omega} a\left|u_{t}^{(\sigma)}(t)\right|^{\beta} u_{t}^{(\sigma)}(t) w_{t}(t+\sigma) d x d t=2 E(u(0)) .
$$

Since we have

$$
\begin{aligned}
& \left.\left|\int_{0}^{\infty} \int_{\Omega} a\right| u_{t}^{(\sigma)}(t)\right|^{\beta} u_{t}^{(\sigma)}(t) w_{t}(t+\sigma) d x d t \mid \\
\leq & \left(\int_{0}^{\infty} \int_{\Omega} a\left|u_{t}^{(\sigma)}(t)\right|^{\beta+2} d x d t\right)^{(\beta+1) /(\beta+2)}\left(\int_{\sigma}^{\infty} \int_{\Omega} a\left|w_{t}(t)\right|^{\beta+2} d x d t\right)^{1 /(\beta+2)} \\
\leq & E(u(0))^{(\beta+1) /(\beta+2)}\left(\int_{\sigma}^{\infty} \int_{\Omega} a\left|w_{t}(t)\right|^{\beta+2} d x d t\right)^{1 /(\beta+2)},
\end{aligned}
$$

it follows from (4.12) that

$$
\int_{\sigma}^{\infty} \int_{\Omega} a\left|w_{t}(t)\right|^{\beta+2} d x d t \geq 2^{\beta+2} E(u(0)),
$$

which contradicts (4.11). Thus the energy $E\left(u^{(\sigma)}(t)\right)$ never decays, which completes the proof of Theorem 2.1 (i).

Finally, we shall prove Theorem 2.1 (ii), i.e., the energy nondecay in the case when $0 \leq \delta \leq 1$. For this, we need the following uniform decay estimate.

Proposition 4.3 (Shibata and Tsutsumi [19]). Let $N \geq 3$. Assume that $\mathbb{R}^{N} \backslash \Omega$ is convex if $\Omega \neq \mathbb{R}^{N}$. Let the data $u_{0}$, $u_{1}$ satisfy $\left\{u_{0}, u_{1}\right\} \in\left[H^{k}(\Omega) \cap W^{k+1,1}(\Omega)\right] \times$ 
$\left[H^{k-1}(\Omega) \cap W^{k, 1}(\Omega)\right]$ with $k=2[N / 2]+3$ and the compatibility condition of order $k$. Then the solution $w(t, x)$ to the problem $(\mathrm{P})_{0}$ satisfies the following estimate:

$$
\sup _{t \geq 0}\left\{(1+t)^{(N-1) / 2}\left(\left\|w_{t}(t)\right\|_{L^{\infty}}+\|\nabla w(t)\|_{L^{\infty}}\right)\right\} \leq C_{k},
$$

where

$$
C_{k} \equiv C\left(\|w(0)\|_{W^{k+1,1}}+\left\|w_{t}(0)\right\|_{H^{k}}+\|w(0)\|_{W^{k, 1}}+\left\|w_{t}(0)\right\|_{H^{k-1}}\right) .
$$

In particular, if $\Omega$ is the whole space $\mathbb{R}^{N}$ and the data $u_{0}$, $u_{1}$ satisfy $\left\{u_{0}, u_{1}\right\} \in$ $\left[H^{k}\left(\mathbb{R}^{N}\right) \cap W^{k+1,1}\left(\mathbb{R}^{N}\right)\right] \times\left[H^{k-1}\left(\mathbb{R}^{N}\right) \cap W^{k, 1}\left(\mathbb{R}^{N}\right)\right]$ with $k=[N / 2]+2$, then the decay estimate (4.13) is valid even if $N \geq 2$.

Now we see from Proposition 4.3 that there exists a constant $C_{k, \beta} \equiv C_{k}^{\beta+2-\eta}>0$ so that

$$
\int_{0}^{\infty} \int_{\Omega} a\left|w_{t}(t)\right|^{\beta+2} d x d t \leq C_{k, \beta}
$$

for all $\eta$ with

$$
\frac{(N-1)(\beta+2-\eta)}{2}>1 \quad \text { and } \quad 2>\eta>2-\frac{2 \delta}{N} .
$$

In fact, we have

$$
\begin{aligned}
& \int_{0}^{\infty} \int_{\Omega} a\left|w_{t}(t)\right|^{\beta+2} d x d t \\
\leq & \int_{0}^{\infty}\left\|w_{t}(t)\right\|_{L^{\infty}}^{\beta+2-\eta}\left(\int_{\Omega} a\left|w_{t}(t)\right|^{\eta} d x\right) d t \\
\leq & C_{k, \beta} \int_{0}^{\infty}(1+t)^{-(N-1)(\beta+2-\eta) / 2}\left(\int_{\Omega}(1+r)^{-2 \delta /(2-\eta)} d x\right)^{(2-\eta) / 2} \\
& \times\left(\int_{\Omega} w_{t}(t)^{2} d x\right)^{\eta / 2} d t .
\end{aligned}
$$

Since

$$
\beta>\frac{2}{N-1}-\frac{2 \delta}{N},
$$

we can choose $\eta$ to satisfy the condition (4.15). Thus, assertion (ii) in Theorem 2.1 can be proved in the same way as assertion (i). The proof of Theorem 2.1 is now complete.

\section{Proof of Theorem 2.2}

Theorem 2.2 is based on the following local existence of solutions.

Proposition 5.1. Let $N=3$. Assume that a $(t, x)$ belongs to $\mathcal{B}^{3}([0, \infty) \times \bar{\Omega})$ and $m=1,2$ or $m \geq 3$. If the data $\left\{u_{0}, u_{1}\right\}$ belong to $H^{4}(\Omega) \times H^{3}(\Omega)$ and satisfy the compatibility condition of order 3 in the sense of (2.5), then there exist numbers $T \equiv T\left(\left\|\nabla u_{0}\right\|_{H^{3}},\left\|u_{1}\right\|_{H^{3}}\right)>0, M_{j} \equiv M_{j}(T)>0(j=1,2)$ and a unique solution 
$u(t, x)$ of the problem $(\mathrm{P})_{m}$ such that

$$
\begin{aligned}
& \nabla u \in C\left([0, T) ; H^{3}(\Omega)\right), \\
& u_{t} \in \bigcap_{j=0}^{2} C^{j}\left([0, T) ; H^{3-j}(\Omega) \cap H_{0}^{1}(\Omega)\right) \cap C^{3}\left([0, T) ; L^{2}(\Omega)\right), \\
& \sup _{0 \leq t<T}\left\{\|\nabla u(t)\|_{H^{3}}+\sum_{j=0}^{3}\left\|D_{t}^{j+1} u(t)\right\|_{H^{3-j}}\right\} \leq M_{1} \varepsilon_{0}, \\
& \sup _{0 \leq t<T}\left\{\left\|u_{t}(t)\right\|_{L^{\infty}}+\left\|u_{t t}(t)\right\|_{L^{\infty}}+\left\|\nabla u_{t}(t)\right\|_{L^{\infty}}\right\} \leq M_{2} \varepsilon_{0} .
\end{aligned}
$$

The proof can be found in Appendix A.

By a standard argument on the basis of Proposition 5.1, it suffices for the existence of a global classical solution to show that for a given $\varepsilon_{0}>0$, if

$$
\left\|u_{t}(t)\right\|_{L^{\infty}}+\left\|u_{t t}(t)\right\|_{L^{\infty}}+\left\|\nabla u_{t}(t)\right\|_{L^{\infty}}<\varepsilon_{0}
$$

holds for assumed smooth solutions $u(t, x)$ on $[0, T)$, then there exists a certain quantity $C>0$, independent of $\varepsilon_{0}$, such that

$$
\sup _{0 \leq t<T}\left\{\|\nabla u(t)\|_{H^{3}}+\sum_{j=0}^{3}\left\|D_{t}^{j+1} u(t)\right\|_{H^{3-j}}\right\} \leq C \sum_{j=0}^{3} I_{j} .
$$

\subsection{A priori estimates I.}

Proposition 5.2. Let $u(t, x)$ be the solution on $[0, T)$ in the sense of Proposition 5.1 satisfying $\left\|u_{t}(t)\right\|_{L^{\infty}}<\varepsilon_{0}$ on $[0, T)$. Then there exists a constant $C>0$, independent of $\varepsilon_{0}$, such that

$$
\begin{aligned}
& \left\|u_{t t}(t)\right\|+\left\|u_{t}(t)\right\|_{H^{1}}+\|\nabla u(t)\|_{H^{1}}+\left(\int_{0}^{t} \int_{\Omega} a u_{t}^{2 m}\left(\left|\nabla u_{t}\right|^{2}+u_{t t}^{2}\right) d x d \tau\right)^{1 / 2} \\
\leq & C\left(I_{0}+I_{1}\right)
\end{aligned}
$$

for $0 \leq t<T$.

Proof. By the energy identity (1.1) we have

$$
\left\|u_{t}(t)\right\|+\|\nabla u(t)\| \leq 2 I_{0}
$$

for $0 \leq t<T$.

Multiplying the equation in $(\mathrm{P})_{m}$ by $-\Delta u_{t}$ and integrating over $\Omega$, we have

$$
\frac{1}{2} \frac{d}{d t}\left\{\left\|\nabla u_{t}(t)\right\|^{2}+\|\Delta u(t)\|^{2}\right\}+\int_{\Omega} \nabla\left(a u_{t}^{2 m+1}\right) \cdot \nabla u_{t} d x=0 .
$$

Next, differentiating the equation in $(\mathrm{P})_{m}$ with respect to $t$ and multiplying it by $u_{t t}$, we have

$$
\frac{1}{2} \frac{d}{d t}\left\{\left\|u_{t t}(t)\right\|^{2}+\left\|\nabla u_{t}(t)\right\|^{2}\right\}+\int_{\Omega} D_{t}\left(a u_{t}^{2 m+1}\right) \cdot u_{t t} d x=0 .
$$


Then it follows from (5.3) and (5.4) that

$$
\begin{aligned}
& \left\|u_{t t}(t)\right\|^{2}+2\left\|\nabla u_{t}(t)\right\|^{2}+\|\Delta u(t)\|^{2}+\int_{0}^{t} \int_{\Omega}(4 m+2) a u_{t}^{2 m}\left(\left|\nabla u_{t}\right|^{2}+u_{t t}^{2}\right) d x d \tau \\
& \leq\left\|u_{t t}(0)\right\|^{2}+2\left\|\nabla u_{t}(0)\right\|^{2}+\|\Delta u(0)\|^{2} \\
& \quad+2 \int_{0}^{t} \int_{\Omega}\left(|\nabla a|\left|\nabla u_{t}\right|+\left|D_{t} a\right|\left|u_{t t}\right|\right)\left|u_{t}\right|^{2 m+1} d x d \tau .
\end{aligned}
$$

Here, it follows from Hypothesis D and the energy identity (1.1) that the last term in the right-hand side of (5.5) is bounded by

$$
\begin{aligned}
& C \int_{0}^{t} \int_{\Omega} a\left(\left|\nabla u_{t}\right|+\left|u_{t t}\right|\right)\left|u_{t}\right|^{2 m+1} d x d \tau \\
\leq & \varepsilon \int_{0}^{t} \int_{\Omega} a\left(\left|\nabla u_{t}\right|^{2}+u_{t t}^{2}\right) u_{t}^{2 m} d x d \tau+C_{\varepsilon} \int_{0}^{t} \int_{\Omega} a u_{t}^{2 m+2} d x d \tau \\
\leq & \varepsilon \int_{0}^{t} \int_{\Omega} a\left(\left|\nabla u_{t}\right|^{2}+u_{t t}^{2}\right) u_{t}^{2 m} d x d \tau+C_{\varepsilon} I_{0}^{2}
\end{aligned}
$$

for any $\varepsilon>0$. Using the equation in $(\mathrm{P})_{m}$ and $\left\|u_{t}(0)\right\|_{L^{\infty}}<\varepsilon_{0}$, we see that

$$
\|\Delta u(0)\| \leq C\left\{\left\|u_{t t}(0)\right\|+\left\|u_{t}(0)^{2 m+1}\right\|\right\} \leq C\left(I_{0}+I_{1}\right) .
$$

Thus, it follows from (5.5), (5.6) and (5.7) that

$$
\left\|u_{t t}(t)\right\|^{2}+\left\|\nabla u_{t}(t)\right\|^{2}+\|\Delta u(t)\|^{2}+\int_{0}^{t} \int_{\Omega} a u_{t}^{2 m}\left(\left|\nabla u_{t}\right|^{2}+u_{t t}^{2}\right) d x d \tau \leq C\left(I_{0}^{2}+I_{1}^{2}\right) .
$$

This and Lemma 3.2 lead to (5.1).

5.2. $L^{1}$ bounds for the weighted energy. Hereafter we let $\varphi(r)$ be as in (4.10), where $\delta>1,0<\gamma<1$ and $\mu>1$. Recalling the definition of $\boldsymbol{\theta}$ in section 3, we have an $L^{1}$ bound for the weighted energy.

Proposition 5.3. Let $u(t, x)$ be as in Proposition 5.1 satisfying $\left\|u_{t}(t)\right\|_{L^{\infty}}<\varepsilon_{0}$ on $[0, T)$ for small $\varepsilon_{0}$ such that

$$
0<\varepsilon_{0}^{2 m}<\frac{\delta-1}{a_{1} \mu \gamma} .
$$

Then there exists a constant $C>0$, independent of $\varepsilon_{0}$, such that

$$
\int_{0}^{t} \int_{\Omega} a\left(u_{t}^{2}+|\boldsymbol{\theta}|^{2}\right) d x d \tau \leq C I_{0}^{2}
$$

for $0 \leq t<T$.

Proof. The proof can be done along the same lines as in Lemma 4.2. Noting that $N=3$, we apply Lemma 3.3 to obtain

$$
X_{t}+Z=\nabla \cdot Y
$$


where we set

$$
\left\{\begin{aligned}
X & =\frac{1}{2}\left(u_{t}^{2}+|\boldsymbol{\theta}|^{2}\right)+\varphi(r)(\widetilde{x} \cdot \boldsymbol{\theta}) u_{t} \\
\boldsymbol{Y} & =\boldsymbol{\theta} u_{t}+\varphi(r)\left\{(\widetilde{x} \cdot \boldsymbol{\theta}) \boldsymbol{\theta}+\frac{1}{2} \widetilde{x}\left(u_{t}^{2}-|\boldsymbol{\theta}|^{2}\right)\right\} \\
Z & =\frac{1}{2} \varphi^{\prime}(r)\left(u_{t}^{2}+|\boldsymbol{\theta}|^{2}\right)+\left\{\frac{1}{r} \varphi(r)-\varphi^{\prime}(r)\right\}\left\{|\boldsymbol{\theta}|^{2}-(\widetilde{x} \cdot \boldsymbol{\theta})^{2}\right\}+a u_{t}^{2 m+2} \\
& \quad+a \varphi(r)(\widetilde{x} \cdot \boldsymbol{\theta}) u_{t}^{2 m+1}
\end{aligned}\right.
$$

Recalling that $\boldsymbol{Y} \cdot \boldsymbol{\nu} \leq 0$ on $\partial \Omega$ (see (4.8) in the proof of Lemma 4.2), we integrate (5.10) over $(0, t) \times \Omega$ to obtain

$$
\int_{\Omega} X(t, x) d x+\int_{0}^{t} \int_{\Omega} Z(\tau, x) d x d \tau \leq \int_{\Omega} X(0, x) d x .
$$

As in the proof of Lemma 4.2, we have

$$
\sup _{t \geq 0} \int_{\Omega}|X(t, x)| d x \leq C I_{0}^{2} .
$$

Further, since we have $\left(r^{-1} \varphi-\varphi^{\prime}\right)\left\{|\boldsymbol{\theta}|^{2}-(\widetilde{x} \cdot \boldsymbol{\theta})^{2}\right\} \geq 0$ and $\left\|u_{t}(t)\right\|_{L^{\infty}}<\varepsilon_{0}$ on $[0, T)$, it follows from (5.8) that

$$
\begin{aligned}
Z & \geq \frac{1}{2} \varphi^{\prime}(r)\left(u_{t}^{2}+|\boldsymbol{\theta}|^{2}\right)+a\left\{u_{t}^{2 m+2}+\varphi(r)(\widetilde{x} \cdot \boldsymbol{\theta}) u_{t}^{2 m+1}\right\} \\
& =\frac{1}{2} \varphi^{\prime}(r)\left(u_{t}^{2}+|\boldsymbol{\theta}|^{2}\right)+a u_{t}^{2 m}\left\{u_{t}^{2}+\varphi(r)(\widetilde{x} \cdot \boldsymbol{\theta}) u_{t}+\frac{1}{4} \varphi(r)^{2}|\boldsymbol{\theta}|^{2}\right\}-\frac{1}{4} a \varphi(r)^{2} u_{t}^{2 m}|\boldsymbol{\theta}|^{2} \\
& \geq \frac{\gamma(\delta-1)}{2 a_{1} \mu} a\left(u_{t}^{2}+|\boldsymbol{\theta}|^{2}\right)-\frac{\gamma^{2} \varepsilon_{0}^{2 m}}{4} a|\boldsymbol{\theta}|^{2} \\
& >\frac{\gamma(\delta-1)}{4 a_{1} \mu} a\left(u_{t}^{2}+|\boldsymbol{\theta}|^{2}\right) .
\end{aligned}
$$

Therefore, it follows from (5.11)-(5.13) that the inequality (5.9) is valid. This completes the proof of Proposition 5.3.

For the proof of an $L^{1}$ bound for the weighted energy of higher-order derivatives, we prepare an $L^{1}$ bound for the weighted energy of the linearized equation.

Proposition 5.4. Let $u(t, x)$ be the solution in the sense of Proposition 5.1 satisfying

$$
\left\|u_{t}(t)\right\|_{L^{\infty}}+\left\|u_{t t}(t)\right\|_{L^{\infty}}<\varepsilon_{0} \quad \text { on }[0, T),
$$

where we choose $\varepsilon_{0}$ so small that

$$
0<\varepsilon_{0}^{2 m}<\frac{\delta-1}{(2 m+1) a_{1} \mu \gamma} .
$$

Consider the linear problem

$(\mathrm{P})_{l}\left\{\begin{array}{l}U_{t t}^{(l)}-\Delta U^{(l)}+(2 m+1) a(t, x) u_{t}^{2 m} U_{t}^{(l)}=\sigma_{l}\left(a, u_{t}\right), \quad(t, x) \in(0, \infty) \times \Omega, \\ U^{(l)}(0)=U_{0}^{(l)} \in H_{0}^{1}(\Omega), \quad U_{t}^{(l)}(0)=U_{1}^{(l)} \in L^{2}(\Omega), \\ U^{(l)}(t, x)=0,\end{array} \quad(t, x) \in(0, \infty) \times \partial \Omega\right.$, 
for $l=1,2,3$, where we set

$$
\begin{aligned}
\sigma_{1}\left(a, u_{t}\right)= & -a_{t} u_{t}^{2 m+1}, \\
\sigma_{2}\left(a, u_{t}\right)= & -\left(D_{t}^{2} a\right) u_{t}^{2 m+1}-(4 m+2) a_{t} u_{t}^{2 m} D_{t}^{2} u-m(4 m+2) a u_{t}^{2 m-1}\left(D_{t}^{2} u\right)^{2}, \\
\sigma_{3}\left(a, u_{t}\right)= & -\left(D_{t}^{3} a\right) u_{t}^{2 m+1}-(6 m+3)\left(D_{t}^{2} a\right) u_{t}^{2 m} D_{t}^{2} u \\
& -(6 m+3) a_{t}\left\{2 m u_{t}^{2 m-1}\left(D_{t}^{2} u\right)^{2}+u_{t}^{2 m} D_{t}^{3} u\right\} \\
& -2 m(2 m+1) a\left\{(2 m-1) u_{t}^{2 m-2}\left(D_{t}^{2} u\right)^{3}+3 u_{t}^{2 m-1}\left(D_{t}^{2} u\right)\left(D_{t}^{3} u\right)\right\} .
\end{aligned}
$$

Then there exists a constant $C>0$, independent of $\varepsilon_{0}$, such that

$$
\begin{aligned}
& E\left(U^{(l)}(t)\right)+\int_{0}^{t} \int_{\Omega} a\left\{\left|U_{t}^{(l)}\right|^{2}+\left|\nabla U^{(l)}\right|^{2}+\left|\boldsymbol{\theta}^{(l)}\right|^{2}\right\} d x d \tau \\
\leq & C\left\{E\left(U^{(l)}(0)\right)+\left\|U^{(l)}(0)\right\|^{2}+\left\|U^{(l)}(t)\right\|^{2}+\int_{0}^{t} \int_{\Omega} a\left(\left|U^{(l)}\right|^{2}+\sum_{j=1}^{l}\left|D_{t}^{j} u\right|^{2}\right) d x d \tau\right\}
\end{aligned}
$$

for $0 \leq t<T$. Here we set

$$
\boldsymbol{\theta}^{(l)}=\nabla U^{(l)}+\frac{1}{r} \widetilde{x} U^{(l)} .
$$

Proof. First step. We may assume that $U^{(l)}$ is smooth. As in the derivation of the identity (5.10), we have

$$
X_{t}+Z_{1}=\nabla \cdot Y+Z_{2},
$$

where we set

$$
\left\{\begin{aligned}
X= & \frac{1}{2}\left\{\left|U_{t}^{(l)}\right|^{2}+\left|\boldsymbol{\theta}^{(l)}\right|^{2}\right\}+\varphi(r)\left(\widetilde{x} \cdot \boldsymbol{\theta}^{(l)}\right) U_{t}^{(l)} \\
\boldsymbol{Y}= & \boldsymbol{\theta}^{(l)} U_{t}^{(l)}+\varphi(r)\left\{\left(\widetilde{x} \cdot \boldsymbol{\theta}^{(l)}\right) \boldsymbol{\theta}^{(l)}+\frac{1}{2} \widetilde{x}\left(\left|U_{t}^{(l)}\right|^{2}-\left|\boldsymbol{\theta}^{(l)}\right|^{2}\right)\right\} \\
Z_{1}= & \frac{1}{2} \varphi^{\prime}(r)\left\{\left|U_{t}^{(l)}\right|^{2}+\left|\boldsymbol{\theta}^{(l)}\right|^{2}\right\}+\left\{\frac{1}{r} \varphi(r)-\varphi^{\prime}(r)\right\}\left\{\left|\boldsymbol{\theta}^{(l)}\right|^{2}-\left(\widetilde{x} \cdot \boldsymbol{\theta}^{(l)}\right)^{2}\right\} \\
& \quad+(2 m+1) a u_{t}^{2 m}\left|U_{t}^{(l)}\right|^{2}+(2 m+1) a \varphi(r) u_{t}^{2 m} U_{t}^{(l)}\left(\widetilde{x} \cdot \boldsymbol{\theta}^{(l)}\right), \\
Z_{2}= & \sigma_{l}\left(a, u_{t}\right) U_{t}^{(l)}+\varphi \sigma_{l}\left(a, u_{t}\right)\left(\widetilde{x} \cdot \boldsymbol{\theta}^{(l)}\right) .
\end{aligned}\right.
$$

Integrate (5.17) over $(0, t) \times \Omega$. Then

$$
\left.\int_{\Omega} X(\tau, x) d x\right|_{\tau=0} ^{\tau=t}+\int_{0}^{t} \int_{\Omega} Z_{1}(\tau, x) d x d \tau \leq \int_{0}^{t} \int_{\Omega}\left|Z_{2}(\tau, x)\right| d x d \tau
$$

where we have used

$$
\boldsymbol{Y} \cdot \boldsymbol{\nu}=\frac{1}{2} \varphi(\widetilde{x} \cdot \boldsymbol{\nu})\left(\frac{\partial U^{(l)}}{\partial \boldsymbol{\nu}}\right)^{2} \leq 0 \quad \text { on } \partial \Omega .
$$

Since $\varphi(r) \leq \gamma<1$, we see that

$$
\int_{\Omega} X(t, x) d x \geq \int_{\Omega} \frac{1-\gamma}{2}\left\{\left|U_{t}^{(l)}\right|^{2}+\left|\boldsymbol{\theta}^{(l)}\right|^{2}\right\} d x \geq 0,
$$

and it follows from the Hardy inequality (4.7) that

$$
\int_{\Omega}|X(0, x)| d x \leq C E\left(U^{(l)}(0)\right) .
$$


We see from the smallness condition (5.15) on $\varepsilon_{0}$ that

$$
\begin{aligned}
Z_{1} \geq & \frac{1}{2} \varphi^{\prime}(r)\left\{\left|U_{t}^{(l)}\right|^{2}+\left|\boldsymbol{\theta}^{(l)}\right|^{2}\right\} \\
& +(2 m+1) a u_{t}^{2 m}\left\{\left|U_{t}^{(l)}\right|^{2}+\varphi(r)\left(\widetilde{x} \cdot \boldsymbol{\theta}^{(l)}\right) U_{t}^{(l)}\right\} \\
= & \frac{1}{2} \varphi^{\prime}(r)\left\{\left|U_{t}^{(l)}\right|^{2}+\left|\boldsymbol{\theta}^{(l)}\right|^{2}\right\} \\
& +(2 m+1) a u_{t}^{2 m}\left\{\left|U_{t}^{(l)}\right|^{2}+\varphi(r)\left(\widetilde{x} \cdot \boldsymbol{\theta}^{(l)}\right) U_{t}^{(l)}+\frac{1}{4} \varphi(r)^{2}\left|\boldsymbol{\theta}^{(l)}\right|^{2}\right\} \\
& -\frac{2 m+1}{4} a u_{t}^{2 m} \varphi(r)^{2}\left|\boldsymbol{\theta}^{(l)}\right|^{2} \\
\geq & \frac{\gamma(\delta-1)}{2 a_{1} \mu} a\left\{\left|U_{t}^{(l)}\right|^{2}+\left|\boldsymbol{\theta}^{(l)}\right|^{2}\right\}-\frac{(2 m+1) \gamma^{2} \varepsilon_{0}^{2 m}}{4} a\left|\boldsymbol{\theta}^{(l)}\right|^{2} \\
> & \frac{\gamma(\delta-1)}{4 a_{1} \mu} a\left\{\left|U_{t}^{(l)}\right|^{2}+\left|\boldsymbol{\theta}^{(l)}\right|^{2}\right\} .
\end{aligned}
$$

Hence, it follows from the inequalities (5.18)-(5.21) that

$$
\int_{0}^{t} \int_{\Omega} a\left\{\left|U_{t}^{(l)}\right|^{2}+\left|\boldsymbol{\theta}^{(l)}\right|^{2}\right\} d x d \tau \leq C\left\{E\left(U^{(l)}(0)\right)+\int_{0}^{t} \int_{\Omega}\left|Z_{2}(\tau, x)\right| d x d \tau\right\}
$$

for $0 \leq t<T$.

Second step. Multiplying the equation in $(\mathrm{P})_{l}$ by $U_{t}^{(l)}$, we have

$$
\begin{aligned}
& E\left(U^{(l)}(t)\right)+(2 m+1) \int_{0}^{t} \int_{\Omega} a u_{t}^{2 m}\left|U_{t}^{(l)}\right|^{2} d x d \tau \\
\leq & E\left(U^{(l)}(0)\right)+\int_{0}^{t} \int_{\Omega}\left|\sigma_{l}\right|\left|U_{t}^{(l)}\right| d x d \tau .
\end{aligned}
$$

Then it follows from the estimates (5.22) and (15.23) that

$$
\begin{aligned}
& E\left(U^{(l)}(t)\right)+\int_{0}^{t} \int_{\Omega} a u_{t}^{2 m}\left|U_{t}^{(l)}\right|^{2} d x d \tau \\
& +2 \int_{0}^{t} \int_{\Omega} a\left\{\left|U_{t}^{(l)}\right|^{2}+\left|\boldsymbol{\theta}^{(l)}\right|^{2}\right\} d x d \tau \\
\leq & C\left\{E\left(U^{(l)}(0)\right)+\int_{0}^{t} \int_{\Omega}\left|\sigma_{l}\right|\left(\left|U_{t}^{(l)}\right|+\left|\boldsymbol{\theta}^{(l)}\right|\right) d x d \tau\right\} .
\end{aligned}
$$

Further, multiplying the equation in $(\mathrm{P})_{l}$ by $a U^{(l)}$, we have

$$
\begin{aligned}
& \int_{0}^{t} \int_{\Omega} a\left\{\left|\nabla U^{(l)}\right|^{2}-\mid\right.\left.\left.U_{t}^{(l)}\right|^{2}\right\} d x d \tau+(2 m+1) \int_{0}^{t} \int_{\Omega} a^{2} u_{t}^{2 m+1} U_{t}^{(l)} U^{(l)} d x d \tau \\
&+\left.\left(a U^{(l)}(\tau), U_{t}^{(l)}(\tau)\right)\right|_{\tau=0} ^{\tau=t} \\
&=\int_{0}^{t} \int_{\Omega} a_{t} U^{(l)} U_{t}^{(l)} d x d \tau-\int_{0}^{t} \int_{\Omega}\left\{\nabla a \cdot \nabla U^{(l)}\right\} U^{(l)} d x d \tau+\int_{0}^{t} \int_{\Omega} a \sigma_{l} U^{(l)} d x d \tau .
\end{aligned}
$$

Here we can see easily from the energy estimate (5.23) that

$$
\begin{aligned}
& \left|\left(a U^{(l)}(t), U_{t}^{(l)}(t)\right)\right|+\left|\left(a U^{(l)}(0), U_{t}^{(l)}(0)\right)\right| \\
\leq & C\left\{E\left(U^{(l)}(0)\right)+\left\|U^{(l)}(0)\right\|^{2}+\left\|U^{(l)}(t)\right\|^{2}+\int_{0}^{t} \int_{\Omega}\left|\sigma_{l}\right|\left|U_{t}^{(l)}\right| d x d \tau\right\} .
\end{aligned}
$$


Next, noting that

$$
\begin{aligned}
\int_{0}^{t} \int_{\Omega} a_{t} U^{(l)} U_{t}^{(l)} d x d \tau= & \frac{1}{2} \int_{\Omega}\left\{a_{t}(t)\left|U^{(l)}(t)\right|^{2}-a_{t}(0)\left|U^{(l)}(0)\right|^{2}\right\} d x \\
& -\frac{1}{2} \int_{0}^{t} \int_{\Omega} a_{t t}\left|U^{(l)}\right|^{2} d x d \tau,
\end{aligned}
$$

we get

$$
\left|\int_{0}^{t} \int_{\Omega} a_{t} U^{(l)} U_{t}^{(l)} d x d \tau\right| \leq C\left\{\left\|U^{(l)}(0)\right\|^{2}+\left\|U^{(l)}(t)\right\|^{2}+\int_{0}^{t} \int_{\Omega} a\left|U^{(l)}\right|^{2} d x d \tau\right\},
$$

where we have used $\left|a_{t}\right|+\left|a_{t t}\right| \leq C a$. Furthermore, we see from $|\nabla a| \leq C a$ that

$$
\left|\int_{0}^{t} \int_{\Omega}\left\{\nabla a \cdot \nabla U^{(l)}\right\} U^{(l)} d x d \tau\right| \leq \frac{1}{2} \int_{0}^{t} \int_{\Omega} a\left|\nabla U^{(l)}\right|^{2} d x d \tau+C \int_{0}^{t} \int_{\Omega} a\left|U^{(l)}\right|^{2} d x d \tau .
$$

Hence, it follows from the estimates (5.25) -(5.28) that

$$
\begin{aligned}
& \int_{0}^{t} \int_{\Omega} a\left\{\frac{1}{2}\left|\nabla U^{(l)}\right|^{2}-\left|U_{t}^{(l)}\right|^{2}\right\} d x d \tau \\
\leq & C\left\{E\left(U^{(l)}(0)\right)+\left\|U^{(l)}(0)\right\|^{2}+\left\|U^{(l)}(t)\right\|^{2}+\int_{0}^{t} \int_{\Omega} a\left|U^{(l)}\right|^{2} d x d \tau\right. \\
& \left.+\int_{0}^{t} \int_{\Omega} a\left|u_{t}\right|^{2 m+1}\left|U_{t}^{(l)}\right|\left|U^{(l)}\right| d x d \tau+\int_{0}^{t} \int_{\Omega}\left|\sigma_{l}\right|\left(\left|U_{t}^{(l)}\right|+\left|U^{(l)}\right|\right) d x d \tau\right\} .
\end{aligned}
$$

By use of $\left\|u_{t}(t)\right\|_{L^{\infty}}<\varepsilon_{0}$ on $[0, T)$, the fifth term in the right-hand side of (5.29) is estimated as

$$
\begin{aligned}
& C \int_{0}^{t} \int_{\Omega} a\left|u_{t}\right|^{2 m+1}\left|U_{t}^{(l)}\right|\left|U^{(l)}\right| d x d \tau \\
\leq & \frac{\varepsilon}{2} \int_{0}^{t} \int_{\Omega} a u_{t}^{2 m}\left|U_{t}^{(l)}\right|^{2} d x d \tau+C_{\varepsilon} \int_{0}^{t} \int_{\Omega} a u_{t}^{2 m+2}\left|U^{(l)}\right|^{2} d x d \tau \\
\leq & \frac{\varepsilon}{2} \int_{0}^{t} \int_{\Omega} a u_{t}^{2 m}\left|U_{t}^{(l)}\right|^{2} d x d \tau+C_{\varepsilon} \int_{0}^{t} \int_{\Omega} a\left|U^{(l)}\right|^{2} d x d \tau
\end{aligned}
$$

for any $0<\varepsilon<1$ and some $C_{\varepsilon}>0$. Therefore, it follows from the estimates (5.24), (5.29) and (5.30) that

$$
\begin{aligned}
& E\left(U^{(l)}(t)\right)+\int_{0}^{t} \int_{\Omega} a u_{t}^{2 m}\left|U_{t}^{(l)}\right|^{2} d x d \tau \\
& +\int_{0}^{t} \int_{\Omega} a\left\{\left|U_{t}^{(l)}\right|^{2}+\left|\nabla U^{(l)}\right|^{2}+\left|\boldsymbol{\theta}^{(l)}\right|^{2}\right\} d x d \tau \\
\leq & C_{1}\left\{E\left(U^{(l)}(0)\right)+\left\|U^{(l)}(0)\right\|^{2}+\left\|U^{(l)}(t)\right\|^{2}+\int_{0}^{t} \int_{\Omega} a\left|U^{(l)}\right|^{2} d x d \tau\right\} \\
& +C_{2} \int_{0}^{t} \int_{\Omega}\left|\sigma_{l}\right|\left\{\left|U_{t}^{(l)}\right|+\left|U^{(l)}\right|+\left|\boldsymbol{\theta}^{(l)}\right|\right\} d x d \tau .
\end{aligned}
$$

Final step. It suffices for the inequality (5.16) to estimate the last term in the right-hand side of (5.31) in three cases $l=1,2,3$ by using $\sum_{j=1}^{3}\left|D_{t}^{j} a\right| \leq C a$ and $\left\|u_{t}(t)\right\|_{L^{\infty}}+\left\|u_{t t}(t)\right\|_{L^{\infty}}<\varepsilon_{0}$ on $[0, T)$. 
Case $l=1$. In this case

\{the last term in the right-hand side of (5.31) $\}$

$$
\begin{aligned}
& \leq C \int_{0}^{t} \int_{\Omega}\left|a_{t}\right|\left|u_{t}\right|^{2 m+1}\left\{\left|U_{t}^{(1)}\right|+\left|U^{(1)}\right|+\left|\boldsymbol{\theta}^{(1)}\right|\right\} d x d \tau \\
& \leq \frac{\varepsilon}{2} \int_{0}^{t} \int_{\Omega} a\left\{u_{t}^{2 m}\left|U_{t}^{(1)}\right|^{2}+\left|\boldsymbol{\theta}^{(1)}\right|^{2}\right\} d x d \tau+C_{\varepsilon} \int_{0}^{t} \int_{\Omega} a\left\{u_{t}^{2}+\left|U^{(1)}\right|^{2}\right\} d x d \tau
\end{aligned}
$$

for any $0<\varepsilon<1$ and some $C_{\varepsilon}>0$. Therefore, it follows from the estimates (5.31) and (5.32) that (5.16) is valid for $l=1$.

Case $l=2$. In this case

\{the last term in the right-hand side of (5.31) $\}$

$$
\begin{aligned}
\leq & C \int_{0}^{t} \int_{\Omega} a\left\{\left|u_{t}\right|^{2 m+1}+u_{t}^{2 m}\left|D_{t}^{2} u\right|+\left|u_{t}\right|^{2 m-1}\left|D_{t}^{2} u\right|^{2}\right\}\left\{\left|U_{t}^{(2)}\right|+\left|U^{(2)}\right|+\left|\boldsymbol{\theta}^{(2)}\right|\right\} d x d \tau \\
\leq & \frac{\varepsilon}{2} \int_{0}^{t} \int_{\Omega} a\left\{u_{t}^{2 m}\left|U_{t}^{(2)}\right|^{2}+\left|\boldsymbol{\theta}^{(2)}\right|^{2}+\left|U_{t}^{(2)}\right|^{2}\right\} d x d \tau \\
& +C_{\varepsilon} \int_{0}^{t} \int_{\Omega} a\left\{u_{t}^{2 m+2}+u_{t}^{4 m+2}+\left|U^{(2)}\right|^{2}+u_{t}^{2 m}\left|D_{t}^{2} u\right|^{2}+u_{t}^{4 m}\left|D_{t}^{2} u\right|^{2}\right. \\
& \left.\quad+u_{t}^{4 m-2}\left|D_{t}^{2} u\right|^{4}\right\} d x d \tau \\
\leq & \frac{\varepsilon}{2} \int_{0}^{t} \int_{\Omega} a\left\{u_{t}^{2 m}\left|U_{t}^{(2)}\right|^{2}+\left|\boldsymbol{\theta}^{(2)}\right|^{2}+\left|U_{t}^{(2)}\right|^{2}\right\} d x d \tau \\
& +C_{\varepsilon} \int_{0}^{t} \int_{\Omega} a\left\{u_{t}^{2}+\left|D_{t}^{2} u\right|^{2}+\left|U^{(2)}\right|^{2}\right\} d x d \tau
\end{aligned}
$$

for any $0<\varepsilon<1$ and some $C_{\varepsilon}>0$. Therefore, it follows from the estimates (5.31) and (5.33) that (5.16) is valid for $l=2$.

Case $l=3$. In this case

\{the last term in the right-hand side of (5.31) $\}$

$$
\begin{aligned}
& \leq C \int_{0}^{t} \int_{\Omega} a\left\{\left|u_{t}\right|^{2 m+1}+u_{t}^{2 m}\left|D_{t}^{2} u\right|+\left|u_{t}\right|^{2 m-1}\left|D_{t}^{2} u\right|^{2}+u_{t}^{2 m}\left|D_{t}^{3} u\right|+u_{t}^{2 m-2}\left|D_{t}^{2} u\right|^{3}\right. \\
& \left.\quad+\left|u_{t}\right|^{2 m-1}\left|D_{t}^{2} u\right|\left|D_{t}^{3} u\right|\right\}\left\{\left|U_{t}^{(3)}\right|+\left|U^{(3)}\right|+\left|\boldsymbol{\theta}^{(3)}\right|\right\} d x d \tau \\
& \leq \frac{\varepsilon}{2} \int_{0}^{t} \int_{\Omega} a\left\{u_{t}^{2 m}\left|U_{t}^{(3)}\right|^{2}+\left|\boldsymbol{\theta}^{(3)}\right|^{2}+\left|U_{t}^{(3)}\right|^{2}\right\} d x d \tau \\
& \quad+C_{\varepsilon} \int_{0}^{t} \int_{\Omega} a\left\{u_{t}^{2}+\left|D_{t}^{2} u\right|^{2}+\left|D_{t}^{3} u\right|^{2}+\left|U^{(3)}\right|^{2}\right\} d x d \tau
\end{aligned}
$$

for any $0<\varepsilon<1$ and some $C_{\varepsilon}>0$. Therefore, it follows from the estimates (5.31) and (5.34) that (5.16) is valid for $l=3$. This completes the proof of Proposition 5.4 . 
Proposition 5.5. Let $u(t, x)$ be as in Proposition 5.4. Then there exists a constant $C>0$, independent of $\varepsilon_{0}$, such that

$E\left(D_{t}^{l} u(t)\right)+\int_{0}^{t} \int_{\Omega} a\left(\left|D_{t}^{l+1} u\right|^{2}+\left|D_{t}^{l} \nabla u\right|^{2}+\left|D_{t}^{l} \boldsymbol{\theta}\right|^{2}\right) d x d \tau \leq C\left\{\left\|D_{t}^{l} u(t)\right\|^{2}+\sum_{j=0}^{l} I_{j}^{2}\right\}$

for $0 \leq t<T$ and $l=1,2,3$.

Proof. Differentiating the equation in $(\mathrm{P})_{m} l$ times with respect to $t(l=1,2,3)$, we have

$$
D_{t}^{l+2} u-\Delta D_{t}^{l} u+(2 m+1) a u_{t}^{2 m} D_{t}^{l} u=\sigma_{l}\left(a, u_{t}\right) .
$$

Then, setting $U^{(1)}=u_{t}$ in Proposition 5.4, we have (5.35) for $l=1$. Similarly, setting $U^{(l)}=D_{t}^{l} u(l=2,3)$ in Proposition 5.4, we have (5.35) for $l=2,3$, respectively.

\subsection{A priori estimates II.}

Proposition 5.6. In addition to the assumptions in Proposition 5.5, we further assume that $\left\|\nabla u_{t}(t)\right\|_{L^{\infty}}<\varepsilon_{0}$ on $[0, T)$. Then there exists a constant $C>0$, independent of $\varepsilon_{0}$, such that

$$
\begin{gathered}
\left\|u_{t}(t)\right\|_{H^{2}}+\|\nabla u(t)\|_{H^{2}} \leq C\left(I_{0}+I_{1}+I_{2}\right), \\
\left\|u_{t t}(t)\right\|_{H^{1}}+\left\|D_{t}^{3} u(t)\right\| \leq C\left(I_{0}+I_{1}+I_{2}\right)
\end{gathered}
$$

for $0 \leq t<T$.

Proof. Differentiating the equation in $(\mathrm{P})_{m}$ twice with respect to $x_{j}(j=1,2,3)$, we have

$$
(\Delta u)_{t t}-\Delta(\Delta u)+(2 m+1) a u_{t}^{2 m} \Delta u_{t}=\sigma_{\Delta}\left(a, u_{t}\right),
$$

where we set

$$
\sigma_{\Delta}\left(a, u_{t}\right)=-(\Delta a) u_{t}^{2 m+1}-(4 m+2) \nabla a \cdot u_{t}^{2 m} \nabla u_{t}-2 m(2 m+1) a u_{t}^{2 m-1}\left|\nabla u_{t}\right|^{2}
$$

Since $D_{t}^{j} u(t, x)=0$ on $\partial \Omega(j=0,1,2,3)$, we use the equation in $(\mathrm{P})_{m}$ to obtain

$$
\Delta u_{t}=D_{t}^{3} u+a_{t} u_{t}^{2 m+1}+(2 m+1) a u_{t}^{2 m} D_{t}^{2} u=0 \quad \text { on } \partial \Omega .
$$

Noting this and multiplying (5.38) by $\Delta u_{t}$, we have

$$
\begin{aligned}
& E(\Delta u(t))+(2 m+1) \int_{0}^{t} \int_{\Omega} a u_{t}^{2 m}\left|\Delta u_{t}\right|^{2} d x d \tau \\
\leq & E(\Delta u(0))+\int_{0}^{t} \int_{\Omega}\left|\sigma_{\Delta}\right|\left|\Delta u_{t}\right| d x d \tau .
\end{aligned}
$$

In view of Propositions 5.2 and 5.5 for $l=1$, we have proved that

$$
\int_{0}^{t} \int_{\Omega} a\left(u_{t}^{2 m}\left|\nabla u_{t}\right|^{2}+\left|\nabla u_{t}\right|^{2}\right) d x d \tau \leq C\left(I_{0}^{2}+I_{1}^{2}\right),
$$


from which it follows that

$$
\begin{aligned}
& \int_{0}^{t} \int_{\Omega}\left|\sigma_{\Delta}\right|\left|\Delta u_{t}\right| d x d \tau \\
\leq & C \int_{0}^{t} \int_{\Omega} a\left(\left|u_{t}\right|^{2 m+1}+u_{t}^{2 m}\left|\nabla u_{t}\right|+\left|u_{t}\right|^{2 m-1}\left|\nabla u_{t}\right|^{2}\right)\left|\Delta u_{t}\right| d x d \tau \\
\leq & \varepsilon \int_{0}^{t} \int_{\Omega} a u_{t}^{2 m}\left|\Delta u_{t}\right|^{2} d x d \tau \\
& +C_{\varepsilon} \int_{0}^{t} \int_{\Omega} a\left(\left|u_{t}\right|^{2 m+2}+u_{t}^{2 m}\left|\nabla u_{t}\right|^{2}+u_{t}^{2 m-2}\left|\nabla u_{t}\right|^{4}\right) d x d \tau \\
\leq & \varepsilon \int_{0}^{t} \int_{\Omega} a u_{t}^{2 m}\left|\Delta u_{t}\right|^{2} d x d \tau+C \int_{0}^{t} \int_{\Omega} a\left(u_{t}^{2}+u_{t}^{2 m}\left|\nabla u_{t}\right|^{2}+\left|\nabla u_{t}\right|^{2}\right) d x d \tau \\
\leq & \varepsilon \int_{0}^{t} \int_{\Omega} a u_{t}^{2 m}\left|\Delta u_{t}\right|^{2} d x d \tau+C\left(I_{0}^{2}+I_{1}^{2}\right)
\end{aligned}
$$

for any $0<\varepsilon<1$, where we have used $\left\|u_{t}(t)\right\|_{L^{\infty}}+\left\|\nabla u_{t}(t)\right\|_{L^{\infty}}<\varepsilon_{0}$ on $[0, T)$. Hence it follows from (5.40) and (5.41) that

$$
E(\Delta u(t))+2 m \int_{0}^{t} \int_{\Omega} a u_{t}^{2 m}\left|\Delta u_{t}\right|^{2} d x d \tau \leq E(\Delta u(0))+C\left(I_{0}^{2}+I_{1}^{2}\right) .
$$

Here we note that

$$
E(\Delta u(0)) \leq C\left(\left\|u_{1}\right\|_{H^{2}}^{2}+\left\|\nabla u_{0}\right\|_{H^{2}}^{2}\right) .
$$

Proposition 5.2 together with (5.43) implies

$$
\left\|u_{t}(t)\right\|_{H^{2}}+\|\nabla u(t)\|_{H^{2}} \leq C\left(I_{0}+I_{1}+I_{2}\right),
$$

which means the estimate (5.36). Finally, using the equation in $(\mathrm{P})_{m}$ and noting that $\left\|u_{t}(t)\right\|_{L^{\infty}}<\varepsilon_{0}$ on $[0, T)$, we have

$$
\begin{aligned}
\left\|u_{t t}(t)\right\|_{H^{1}} & \leq\|\Delta u(t)\|_{H^{1}}+\left\|a u_{t}^{2 m+1}\right\|_{H^{1}} \\
& \leq C\left\{\|\nabla u(t)\|_{H^{2}}+\left\|u_{t}(t)\right\|_{H^{1}}\right\} \\
& \leq C\left(I_{0}+I_{1}+I_{2}\right)
\end{aligned}
$$

for $0 \leq t<T$. This and Proposition 5.5 imply the estimate (5.37).

\subsection{A priori estimates III.}

Proposition 5.7. Let $u(t, x)$ be as in Proposition 5.6. Then there exists a constant $C>0$, independent of $\varepsilon_{0}$, such that

$$
\begin{gathered}
\left\|D_{t}^{2} u(t)\right\|_{H^{2}}+\left\|D_{t}^{3} u(t)\right\|_{H^{1}}+\left\|D_{t}^{4} u(t)\right\| \leq C\left(I_{0}+I_{1}+I_{2}+I_{3}\right), \\
\left\|u_{t}(t)\right\|_{H^{3}}+\|\nabla u(t)\|_{H^{3}} \leq C\left(I_{0}+I_{1}+I_{2}+I_{3}\right),
\end{gathered}
$$

for $0 \leq t<T$.

Proof. Using the equation in $(\mathrm{P})_{m}$, we have

$$
\Delta u_{t t}=D_{t}^{4} u+D_{t}^{2}\left(a u_{t}^{2 m+1}\right) \text {, }
$$


and hence

$$
\begin{aligned}
\left\|\Delta u_{t t}(t)\right\| \leq & \left\|D_{t}^{4} u(t)\right\|+\left\|D_{t}^{2}\left(a u_{t}^{2 m+1}\right)\right\| \\
\leq & C \sum_{j=0}^{3} I_{j} \\
& \quad+C\left\{\left\|\left(D_{t}^{2} a\right) u_{t}^{2 m+1}\right\|+\left\|a_{t} u_{t}^{2 m} D_{t}^{2} u\right\|+\left\|a u_{t}^{2 m-1}\left(D_{t}^{2} u\right)^{2}\right\|+\left\|u_{t}^{2 m} D_{t}^{3} u\right\|\right\} \\
\leq & C \sum_{j=0}^{3} I_{j}+C \varepsilon_{0}^{2 m}\left\{\left\|u_{t}(t)\right\|+\left\|D_{t}^{2} u(t)\right\|+\left\|D_{t}^{3} u(t)\right\|\right\} \\
\leq & C\left(I_{0}+I_{1}+I_{2}+I_{3}\right)
\end{aligned}
$$

for $0 \leq t<T$. Thus, it follows from Proposition 5.5 and (5.46) that the estimate (5.44) is valid.

On the other hand, using the equation in $(\mathrm{P})_{m}$ and $\left\|u_{t}(t)\right\|_{L^{\infty}}+\left\|u_{t t}(t)\right\|_{L^{\infty}}+$ $\left\|\nabla u_{t}(t)\right\|_{L^{\infty}}<\varepsilon_{0}$ on $[0, T)$, we see from (5.44) that

$$
\left\|\Delta u_{t}(t)\right\|_{H^{1}} \leq\left\|D_{t}^{3} u(t)\right\|_{H^{1}}+\left\|D_{t}\left(a u_{t}^{2 m+1}\right)\right\|_{H^{1}} \leq C\left(I_{0}+I_{1}+I_{2}+I_{3}\right)
$$

for $0 \leq t<T$. Hence, again combining (5.47) with Lemma 3.1, we have

$$
\left\|u_{t}(t)\right\|_{H^{3}} \leq C\left\{\left\|\Delta u_{t}(t)\right\|_{H^{1}}+\left\|u_{t}(t)\right\|\right\} \leq C\left(I_{0}+I_{1}+I_{2}+I_{3}\right)
$$

for $0 \leq t<T$.

Finally, it follows from Proposition 5.6 and Lemma 3.2 that

$$
\begin{aligned}
\|\nabla u(t)\|_{H^{3}} & \leq C\left\{I_{0}+I_{1}+I_{2}+\sum_{|\alpha|,|\beta|=2}\left\|D_{x}^{\alpha} D_{x}^{\beta} u(t)\right\|\right\} \\
& \leq C\left\{I_{0}+I_{1}+I_{2}+\sum_{|\alpha|=2}\left\|D_{x}^{\alpha} \Delta u(t)\right\|+\sum_{|\alpha|=2}\left\|D_{x}^{\alpha} \nabla u(t)\right\|\right\} \\
& \leq C\left\{I_{0}+I_{1}+I_{2}+\sum_{|\alpha|=2}\left\|D_{x}^{\alpha} \Delta u(t)\right\|\right\}
\end{aligned}
$$

for $0 \leq t<T$. Here, using the equation in $(\mathrm{P})_{m}$, we see from (5.44) and Proposition 5.6 that

$$
\begin{aligned}
\sum_{|\alpha|=2}\left\|D_{x}^{\alpha} \Delta u(t)\right\| & \leq \sum_{|\alpha|=2}\left\|D_{x}^{\alpha} D_{t}^{2} u(t)\right\|+\sum_{|\alpha|=2}\left\|D_{x}^{\alpha}\left(a u_{t}^{2 m+1}\right)\right\| \\
& \leq C\left\{\left\|D_{t}^{2} u(t)\right\|_{H^{2}}+\left\|u_{t}(t)\right\|_{H^{2}}\right\} \\
& \leq C\left(I_{0}+I_{1}+I_{2}+I_{3}\right)
\end{aligned}
$$

for $0 \leq t<T$. Therefore, combining this with (5.48), we obtain the estimate (5.45).

5.5. Proof of Theorem 2.2 (i) completed. Let $u(t, x)$ be the approximate solution satisfying

$$
\left\|u_{t}(t)\right\|_{L^{\infty}}+\left\|u_{t t}(t)\right\|_{L^{\infty}}+\left\|\nabla u_{t}(t)\right\|_{L^{\infty}}<\varepsilon_{0}
$$

on $[0, T)$. Since the quantity $I_{0}+I_{1}+I_{2}+I_{3}$ is bounded by

$$
C\left\{\left\|\nabla u_{0}\right\|_{H^{3}}+\sum_{j=0}^{3}\left\|D_{t}^{j+1} u(0)\right\|_{H^{3-j}}\right\}
$$


for some $C>0$, it follows from Propositions 5.2, 5.5, 5.6, 5.7 and Sobolev's inequality that

$$
\begin{aligned}
& \|\nabla u(t)\|_{H^{3}}+\sum_{j=0}^{3}\left\|D_{t}^{j+1} u(t)\right\|_{H^{3-j}} \\
\leq & C\left(I_{0}+I_{1}+I_{2}+I_{3}\right) \\
\leq & C_{1}\left\{\left\|\nabla u_{0}\right\|_{H^{3}}+\sum_{j=0}^{3}\left\|D_{t}^{j+1} u(0)\right\|_{H^{3-j}}\right\},
\end{aligned}
$$

and

$$
\begin{aligned}
& \left\|u_{t}(t)\right\|_{L^{\infty}}+\left\|u_{t t}(t)\right\|_{L^{\infty}}+\left\|\nabla u_{t}(t)\right\|_{L^{\infty}} \\
\leq & C\left\{\left\|u_{t}(t)\right\|_{H^{3}}+\left\|u_{t t}(t)\right\|_{H^{2}}\right\} \leq C\left(I_{0}+I_{1}+I_{2}+I_{3}\right) \\
\leq & C_{2}\left\{\left\|\nabla u_{0}\right\|_{H^{3}}+\sum_{j=0}^{3}\left\|D_{t}^{j+1} u(0)\right\|_{H^{3-j}}\right\}
\end{aligned}
$$

on $[0, T)$ for some $C_{j}>0(j=1,2)$. We assume that the initial data $\left\{u_{0}, u_{1}\right\}$ are so small that

$$
\begin{gathered}
\left\|\nabla u_{0}\right\|_{H^{3}}+\sum_{j=0}^{3}\left\|D_{t}^{j+1} u(0)\right\|_{H^{3-j}}<C_{0} \varepsilon_{0}, \\
\left\|u_{t}(0)\right\|_{L^{\infty}}+\left\|u_{t t}(0)\right\|_{L^{\infty}}+\left\|\nabla u_{t}(0)\right\|_{L^{\infty}}<\varepsilon_{0},
\end{gathered}
$$

where we set $C_{0} \equiv C_{2}^{-1}$.

The $a$ priori estimates obtained earlier now show that the local solution $u(t, x)$ can be continued on $[0, \infty)$ under the assumptions (5.49) and (5.50), and the extended solution, denoted by the same, satisfies

$$
\begin{aligned}
& \nabla u \in L^{\infty}\left(0, \infty ; H^{3}(\Omega)\right), \\
& u_{t} \in \bigcap_{j=0}^{2} W^{j, \infty}\left(0, \infty ; H^{3-j}(\Omega) \cap H_{0}^{1}(\Omega)\right) \cap W^{3, \infty}\left(0, \infty ; L^{2}(\Omega)\right), \\
& \underset{\text { ess. } \sup }{t \geq 0}\left\{\|\nabla u(t)\|_{H^{3}}+\sum_{j=0}^{3}\left\|D_{t}^{j+1} u(t)\right\|_{H^{3-j}}\right\} \leq C_{0} C_{1} \varepsilon_{0}, \\
& \quad \text { ess. } \sup \left\{\left\|u_{t}(t)\right\|_{L^{\infty}}+\left\|u_{t t}(t)\right\|_{L^{\infty}}+\left\|\nabla u_{t}(t)\right\|_{L^{\infty}}\right\} \leq \varepsilon_{0} .
\end{aligned}
$$

For completeness we prove that the approximate solution $u(t)$ becomes the strong solution. In fact, by the standard argument, the limit function $u(t)$ satisfies

$$
\begin{aligned}
& \nabla u \in C_{w}\left([0, \infty) ; H^{3}(\Omega)\right), \\
& u_{t} \in \bigcap_{j=0}^{2} \mathcal{B}^{j}\left([0, \infty) ; H^{3-j}(\Omega) \cap H_{0}^{1}(\Omega)\right) \cap C_{w}^{3}\left([0, \infty) ; L^{2}(\Omega)\right) .
\end{aligned}
$$

Let $T>0$ be any fixed number. Then we find that $u(t)$ satisfies

$$
\left(u_{t t}(t), \varphi\right)-(\Delta u(t), \varphi)+\left(a u_{t}(t)^{2 m+1}, \varphi\right)=0 \quad \text { for any } \varphi \in L^{2}(\Omega),
$$


for $0 \leq t \leq T$. On the other hand, we have $a u_{t}^{2 m+1} \in C\left([0, T] ; H_{0}^{1}(\Omega)\right)$. Hence, the general theory of hyperbolic equations (cf. [8, Mizohata, Chapter 6, Theorem 6.12]) implies that the initial-boundary value problem

$$
\begin{cases}u_{t t}^{*}-\Delta u^{*}+a(t, x) u_{t}^{2 m+1}=0, & (t, x) \in(0, \infty) \times \Omega, \\ u^{*}(0, x)=u_{0}(x), \quad u_{t}^{*}(0, x)=u_{1}(x), & x \in \Omega, \\ u^{*}(t, x)=0, & (t, x) \in(0, \infty) \times \partial \Omega\end{cases}
$$

has a unique solution $u^{*}$ satisfying

$$
\begin{aligned}
& \nabla u^{*} \in \mathcal{B}\left([0, \infty) ; H^{3}(\Omega)\right), \\
& u_{t}^{*} \in \bigcap_{j=0}^{2} \mathcal{B}^{j}\left([0, \infty) ; H^{3-j}(\Omega) \cap H_{0}^{1}(\Omega)\right) \cap \mathcal{B}^{3}\left([0, \infty) ; L^{2}(\Omega)\right) .
\end{aligned}
$$

Put $w=u-u^{*}$. Then $w$ satisfies the initial-boundary value problem

$$
\begin{cases}\left(w_{t t}, \varphi\right)-(\Delta w, \varphi)=0, & (t, x) \in(0, \infty) \times \Omega \\ w(0, x)=w_{t}(0, x)=0, & x \in \Omega \\ w(t, x)=0, & (t, x) \in(0, \infty) \times \partial \Omega\end{cases}
$$

for any $\varphi \in L^{2}(\Omega)$. Setting $\varphi=w_{t}$ and integrating over $[0, T]$, we have

$$
\left\|w_{t}(t)\right\|^{2}+\|\nabla w(t)\|^{2}=0,
$$

which implies $w \equiv 0$. Thus $u$ has the regularity in (5.51) and satisfies

$$
\begin{gathered}
\sup _{t \geq 0}\left\{\|\nabla u(t)\|_{H^{3}}+\sum_{j=0}^{3}\left\|D_{t}^{j+1} u(t)\right\|_{H^{3-j}}\right\} \leq C_{0} \varepsilon_{0}, \\
\sup _{t \geq 0}\left\{\left\|u_{t}(t)\right\|_{L^{\infty}}+\left\|u_{t t}(t)\right\|_{L^{\infty}}+\left\|\nabla u_{t}(t)\right\|_{L^{\infty}}\right\} \leq \varepsilon_{0} .
\end{gathered}
$$

The proof of Theorem 2.2 (i) is now complete.

5.6. The decay estimate in the case when $\Omega=\mathbb{R}^{3}$. We consider the Cauchy problem

$$
(\mathrm{P})_{f} \quad \begin{cases}v_{t t}-\Delta v=f, & (t, x) \in(0, \infty) \times \mathbb{R}^{3}, \\ v(0, x)=v_{0}(x), \quad v_{t}(0, x)=v_{1}(x), & x \in \mathbb{R}^{3}\end{cases}
$$

Then the following uniform decay estimate is well known.

Proposition 5.8 (Shibata and Tsutsumi [19). Let $v(t, x)$ be the solution of the problem $(\mathrm{P})_{f}$ with the data $\left\{v_{0}, v_{1}\right\} \in\left[W^{4,1}\left(\mathbb{R}^{3}\right) \cap H^{3}\left(\mathbb{R}^{3}\right)\right] \times\left[W^{3,1}\left(\mathbb{R}^{3}\right) \cap H^{2}\left(\mathbb{R}^{3}\right)\right]$ and $|f|_{1,1+\eta, 3}+|f|_{2,1+\eta, 2}<\infty$ for any $\eta>0$, where we set

$$
|f|_{p, q, k}=\sup _{t \geq 0}\left\{(1+t)^{q} \sum_{j+|\alpha| \leq k}\left\|D_{t}^{j} D_{x}^{\alpha} f(t, \cdot)\right\|_{L^{p}}\right\} .
$$

Then $v(t, x)$ satisfies

$$
\begin{aligned}
& \sup _{t \geq 0}\left\{(1+t)\left(\left\|v_{t}(t)\right\|_{L^{\infty}}+\|\nabla v(t)\|_{L^{\infty}}\right)\right\} \\
\leq & C\left(\left\|v_{0}\right\|_{W^{4,1}}+\left\|v_{0}\right\|_{H^{3}}+\left\|v_{1}\right\|_{W^{3,1}}+\left\|v_{1}\right\|_{H^{2}}+|f|_{1,1+\eta, 3}+|f|_{2,1+\eta, 2}\right) .
\end{aligned}
$$


We will now combine Theorem 2.2 (i) with Proposition 5.8 to prove Theorem 2.2 (ii). Since we can regard $f$ as $-a u_{t}^{2 m+1}$ in Proposition 5.8, we have

$$
\begin{aligned}
& \sup _{t \geq 0}\left\{(1+t)\left(\left\|u_{t}(t)\right\|_{L^{\infty}}+\|\nabla u(t)\|_{L^{\infty}}\right)\right\} \\
\leq & C\left(\left\|u_{0}\right\|_{W^{4,1}}+\left\|u_{0}\right\|_{H^{3}}+\left\|u_{1}\right\|_{W^{3,1}}+\left\|u_{1}\right\|_{H^{2}}\right. \\
& \left.+\left|a u_{t}^{2 m+1}\right|_{1,1+\eta, 3}+\left|a u_{t}^{2 m+1}\right|_{2,1+\eta, 2}\right) .
\end{aligned}
$$

Let us estimate the last two terms in the right-hand side of (5.53),

$$
(1+t)^{1+\eta} \sum_{j+|\alpha| \leq 3}\left\|D_{t}^{j} D_{x}^{\alpha}\left(a u_{t}^{2 m+1}\right)\right\|_{L^{1}} \text { and }(1+t)^{1+\eta} \sum_{j+|\alpha| \leq 2}\left\|D_{t}^{j} D_{x}^{\alpha}\left(a u_{t}^{2 m+1}\right)\right\|_{L^{2}} .
$$

For this, we shall use the Sobolev imbedding theorem $H^{2}\left(\mathbb{R}^{3}\right) \subset L^{p}\left(\mathbb{R}^{3}\right)(p \geq$ 2 ), Hypothesis D, the uniform bounds of $u_{t}(t)$ in Theorem 2.2 (i) and the decay condition (2.10) of $a(t, x)$.

Case $j+|\alpha|=0$. In this case

$$
\begin{aligned}
(1+t)^{1+\eta}\left\|a u_{t}^{2 m+1}\right\|_{L^{1}} & \leq C(1+t)\left\|u_{t}(t)\right\|_{L^{2 m}}^{2 m}\left\|u_{t}(t)\right\|_{L^{\infty}} \\
& \leq C(1+t)\left\|u_{t}(t)\right\|_{H^{2}}^{2 m}\left\|u_{t}(t)\right\|_{L^{\infty}} \\
& \leq C \varepsilon_{0}^{2 m}(1+t)\left\|u_{t}(t)\right\|_{L^{\infty}}
\end{aligned}
$$

and

$$
\begin{aligned}
(1+t)^{1+\eta}\left\|a u_{t}^{2 m+1}\right\|_{L^{2}} & \leq C(1+t)\left\|u_{t}(t)\right\|_{L^{4 m}}^{2 m}\left\|u_{t}(t)\right\|_{L^{\infty}} \\
& \leq C(1+t)\left\|u_{t}(t)\right\|_{H^{2}}^{2 m}\left\|u_{t}(t)\right\|_{L^{\infty}} \\
& \leq C \varepsilon_{0}^{2 m}(1+t)\left\|u_{t}(t)\right\|_{L^{\infty}} .
\end{aligned}
$$

Case $j+|\alpha|=1$. In this case

$$
\begin{aligned}
& \sum_{j+|\alpha|=1}(1+t)^{1+\eta}\left\|D_{t}^{j} D_{x}^{\alpha}\left(a u_{t}^{2 m+1}\right)\right\|_{L^{1}} \\
\leq & C(1+t) \int_{\mathbb{R}^{3}}\left(\left|u_{t}\right|^{2 m+1}+u_{t}^{2 m}\left|D_{t}^{2} u\right|+u_{t}^{2 m}\left|\nabla u_{t}\right|\right) d x \\
\leq & C(1+t)\left\|u_{t}(t)\right\|_{L^{\infty}} \\
& \times\left\{\left\|u_{t}(t)\right\|_{L^{2 m}}^{2 m}+\left\|u_{t}(t)\right\|_{L^{4 m-2}}^{2 m-1}\left\|D_{t}^{2} u(t)\right\|+\left\|u_{t}(t)\right\|_{L^{4 m-2}}^{2 m-1}\left\|\nabla u_{t}(t)\right\|\right\} \\
\leq & C \varepsilon_{0}^{2 m}(1+t)\left\|u_{t}(t)\right\|_{L^{\infty}}
\end{aligned}
$$

and

$$
\begin{aligned}
& \sum_{j+|\alpha|=1}(1+t)^{1+\eta}\left\|D_{t}^{j} D_{x}^{\alpha}\left(a u_{t}^{2 m+1}\right)\right\|_{L^{2}} \\
\leq & C(1+t)\left\{\int_{\mathbb{R}^{3}}\left(\left|u_{t}\right|^{2 m+1}+u_{t}^{2 m}\left|D_{t}^{2} u\right|+u_{t}^{2 m}\left|\nabla u_{t}\right|\right)^{2} d x\right\}^{1 / 2} \\
\leq & C(1+t)\left\|u_{t}(t)\right\|_{L^{\infty}}^{2 m}\left\{\left\|u_{t}(t)\right\|+\left\|D_{t}^{2} u(t)\right\|+\left\|\nabla u_{t}(t)\right\|\right\} \\
\leq & C \varepsilon_{0}^{2 m}(1+t)\left\|u_{t}(t)\right\|_{L^{\infty}} .
\end{aligned}
$$


Case $j+|\alpha|=2$. In this case

$$
\begin{aligned}
& \sum_{j+|\alpha|=2}(1+t)^{1+\eta}\left\|D_{t}^{j} D_{x}^{\alpha}\left(a u_{t}^{2 m+1}\right)\right\|_{L^{1}} \\
\leq & C(1+t)\left\|u_{t}(t)\right\|_{L^{\infty}} \int_{\mathbb{R}^{3}}\left(u_{t}^{2 m}+u_{t}^{2 m-1}\left|D_{t}^{2} u\right|+\left|u_{t}\right|^{2 m-2}\left|D_{t}^{2} u\right|^{2}+u_{t}^{2 m-1}\left|D_{t}^{3} u\right|\right) d x \\
\leq & C(1+t)\left\|u_{t}(t)\right\|_{L^{\infty}} \\
& \times\left\{\left\|u_{t}(t)\right\|_{L^{2 m}}^{2 m}+\left\|u_{t}(t)\right\|_{L^{4 m-2}}^{2 m-1}\left\|D_{t}^{2} u(t)\right\|\right. \\
& \left.+\left\|u_{t}(t)\right\|_{L^{4 m-4}}^{2 m-2}\left\|D_{t}^{2} u(t)\right\|_{L^{4}}^{2}+\left\|u_{t}(t)\right\|_{L^{4 m-2}}^{2 m-1}\left\|D_{t}^{3} u(t)\right\|\right\} \\
\leq & C \varepsilon_{0}^{2 m}(1+t)\left\|u_{t}(t)\right\|_{L^{\infty}}
\end{aligned}
$$

and

$$
\begin{aligned}
& \sum_{j+|\alpha|=2}(1+t)^{1+\eta}\left\|D_{t}^{j} D_{x}^{\alpha}\left(a u_{t}^{2 m+1}\right)\right\|_{L^{2}} \\
\leq & C(1+t)\left\{\left\|u_{t}(t)\right\|_{L^{\infty}}^{2 m}+\left\|u_{t}(t)\right\|_{L^{\infty}}^{2 m-1}\left\|D_{t}^{2} u(t)\right\|_{L^{\infty}}+\left\|u_{t}(t)\right\|_{L^{\infty}}^{2 m-1}\left\|\nabla u_{t}(t)\right\|_{L^{\infty}}\right\} \\
& \times\left\{\sum_{j=1}^{3}\left\|D_{t}^{j} u(t)\right\|+\sum_{|\alpha|=1}^{2}\left\|D_{t} D_{x}^{\alpha} u(t)\right\|\right\} \\
\leq & C \varepsilon_{0}^{2 m}(1+t)\left\|u_{t}(t)\right\|_{L^{\infty}} .
\end{aligned}
$$

Case $j+|\alpha|=3$. In this case

$$
\sum_{j+|\alpha|=3}(1+t)^{1+\eta}\left\|D_{t}^{j} D_{x}^{\alpha}\left(a u_{t}^{2 m+1}\right)\right\|_{L^{1}} \leq C(1+t)\left\|u_{t}(t)\right\|_{L^{\infty}} \int_{\mathbb{R}^{3}} g\left(\Lambda u_{t}\right) d x
$$

where we set $\Lambda u_{t}=\left(D_{t}^{j} u_{t}, D_{x}^{\alpha} u_{t} ; j=0,1,2,3,|\alpha| \leq 3\right)$ and

$$
\begin{aligned}
g\left(\Lambda u_{t}\right)= & u_{t}^{2 m}+\left|u_{t}\right|^{2 m-1}\left|D_{t}^{2} u\right|+\left|u_{t}\right|^{2 m-2}\left|D_{t}^{2} u\right|^{2}+\left|u_{t}\right|^{2 m-1}\left|D_{t}^{3} u\right|+\left|u_{t}\right|^{2 m-3}\left|D_{t}^{2} u\right|^{3} \\
& +u_{t}^{2 m-2}\left|D_{t}^{2} u\right|\left|D_{t}^{3} u\right|+\left|u_{t}\right|^{2 m-1}\left|D_{t}^{4} u\right| \\
& +\left|u_{t}\right|^{2 m-1}\left|\nabla u_{t}\right|+u_{t}^{2 m-2}\left|\nabla u_{t}\right|\left|D_{t}^{2} u\right|+\left|u_{t}\right|^{2 m-1}\left|\nabla D_{t}^{2} u\right| \\
& +\left|u_{t}\right|^{2 m-3}\left|\nabla u_{t}\right|\left|D_{t}^{2} u\right|^{2}+u_{t}^{2 m-2}\left|D_{t}^{2} u\right|\left|\nabla D_{t}^{2} u\right|+u_{t}^{2 m-2}\left|\nabla u_{t}\right|\left|D_{t}^{3} u\right| \\
& +\left|u_{t}\right|^{2 m-1}\left|\nabla D_{t}^{3} u\right|+u_{t}^{2 m-2}\left|\nabla u_{t}\right|^{2}+\left|u_{t}\right|^{2 m-1}\left|\partial^{2} u_{t}\right| \\
& +\left|u_{t}\right|^{2 m-3}\left|\nabla u_{t}\right|^{2}\left|D_{t}^{2} u\right| \\
& +u_{t}^{2 m-2}\left|\partial^{2} u_{t}\right|\left|D_{t}^{2} u\right|+u_{t}^{2 m-2}\left|\nabla u_{t}\right|\left|\nabla D_{t}^{2} u\right|+\left|u_{t}\right|^{2 m-1}\left|\partial^{2} D_{t}^{2} u\right| \\
& +\left|u_{t}\right|^{2 m-3}\left|\nabla u_{t}\right|^{3}+\left.u_{t}^{2 m-2}\left|\partial^{2} u_{t}\right| \nabla u_{t}|+| u_{t}\right|^{2 m-1}\left|\partial^{3} u_{t}\right| \\
& +u_{t}^{2 m-1}\left|\nabla u_{t}\right|+u_{t}^{2 m-2}\left|\nabla u_{t}\right|^{2}+u_{t}^{2 m-1}\left|\Delta u_{t}\right| .
\end{aligned}
$$

Since $m=2$ or $m \geq 3$, by the same argument as in the previous cases we have

$$
\int_{\mathbb{R}^{3}} g\left(\Lambda u_{t}\right) d x \leq C \varepsilon_{0}^{2 m}
$$


which implies

$$
\sum_{j+|\alpha|=3}(1+t)^{1+\eta}\left\|D_{t}^{j} D_{x}^{\alpha}\left(a u_{t}^{2 m+1}\right)\right\|_{L^{1}} \leq C \varepsilon_{0}^{2 m}(1+t)\left\|u_{t}(t)\right\|_{L^{\infty}} .
$$

Combining the estimate (5.53) and the above four cases, we have

$$
\begin{aligned}
& \sup _{t \geq 0}\left\{(1+t)\left(\left\|u_{t}(t)\right\|_{L^{\infty}}+\|\nabla u(t)\|_{L^{\infty}}\right)\right\} \\
\leq & C\left\{\left\|u_{0}\right\|_{W^{4,1}}+\left\|u_{0}\right\|_{H^{3}}+\left\|u_{1}\right\|_{W^{3,1}}+\left\|u_{1}\right\|_{H^{2}}+\varepsilon_{0}^{2 m} \sup _{t \geq 0}\left((1+t)\left\|u_{t}(t)\right\|_{L^{\infty}}\right)\right\} .
\end{aligned}
$$

If we choose $\varepsilon_{0}$ sufficiently small, we can obtain the desired decay estimate (2.11), which completes the proof of Theorem 2.2.

\section{Proof of Theorem 2.3}

We can prove the assertion by the well-established argument as in 12]. Let $U_{0}(t)$, $t \in \mathbb{R}$, be the unitary group in the energy space $E$ which represents the solution $w(t, x)$ to the problem $(\mathrm{P})_{0}$ with the data $\boldsymbol{f} \equiv\left\{w_{0}, w_{1}\right\} \in E$;

$$
\left\{w(t), w_{t}(t)\right\}=U_{0}(t) \boldsymbol{f} .
$$

Then it follows from Lemma 4.1 for $u^{(\sigma)}(t)$ and $w(t+\sigma)$ replaced by $u(t)$ and $w(t)$, respectively, that

$$
\left(U_{0}(-t) \boldsymbol{u}(t)-U_{0}(-s) \boldsymbol{u}(s), \boldsymbol{f}\right)_{E}=-\int_{s}^{t} \int_{\Omega} a u_{t}^{2 m+1} w_{t} d x d \tau
$$

for any $0 \leq s<t$, where $\boldsymbol{u}(t)$ stands for the pair $\left\{u(t), u_{t}(t)\right\}$. By the $L^{\infty}$ estimate (2.9), the Schwarz inequality and the estimate (4.3) (which is also valid even if the data belongs to $E$ ) appearing in the proof of Lemma 4.2, we have

$$
\left|\left(U_{0}(-t) \boldsymbol{u}(t)-U_{0}(-s) \boldsymbol{u}(s), \boldsymbol{f}\right)_{E}\right| \leq C \varepsilon_{0}^{m}\|\boldsymbol{f}\|_{E}\left(\int_{s}^{t} \int_{\Omega} a u_{t}^{2 m+2} d x d \tau\right)^{1 / 2},
$$

which implies

$$
\left\|U_{0}(-t) \boldsymbol{u}(t)-U_{0}(-s) \boldsymbol{u}(s)\right\|_{E} \rightarrow 0 \quad \text { as } s, t \rightarrow \infty,
$$

and $U_{0}(-t) \boldsymbol{u}(t)$ converges in $E$ as $t \rightarrow \infty$. Put

$$
f^{+} \equiv\left\{u_{0}^{+}, u_{1}^{+}\right\}=\underset{t \rightarrow \infty}{\mathrm{s}-\lim _{0}} U_{0}(-t) \boldsymbol{u}(t) .
$$

Then $f^{+} \in E$, and we have

$$
\left\|\boldsymbol{u}(t)-U_{0}(t) \boldsymbol{f}^{+}\right\|_{E}=\left\|U_{0}(-t) \boldsymbol{u}(t)-\boldsymbol{f}^{+}\right\|_{E} \rightarrow 0 \quad \text { as } t \rightarrow \infty .
$$

The proof is complete.

\section{Proof of Theorem 2.4}

Let $U_{0}(t)$ be as in the previous section. By Duhamel's principle the equation in $(\mathrm{P})_{m}$ can be written as an integral equation of the form

$$
\boldsymbol{u}(t)=U_{0}(t) \boldsymbol{f}+\int_{0}^{t} U_{0}(t-\tau) \boldsymbol{F}\left(u_{t}(\tau)\right) d \tau,
$$

where we set

$$
\boldsymbol{u}(t)=\left\{u(t), u_{t}(t)\right\}, \quad f=\left\{u_{0}, u_{1}\right\} \quad \text { and } \quad \boldsymbol{F}\left(u_{t}(t)\right)=\left\{0,-a u_{t}(t)^{2 m+1}\right\} .
$$


It is well known from Morawetz [13] (cf. Nakao [16]) that if $f \in E$ has a compact support in $B_{R}$, then we have

$$
\left\|U_{0}(t) \boldsymbol{f}\right\|_{E_{R}} \leq C(R) \mathrm{e}^{-\lambda t}\|\boldsymbol{f}\|_{E}
$$

for some $\lambda>0$, independent of $R$, and $C(R) \equiv e^{\lambda R}$, where we set

$$
\|\boldsymbol{f}\|_{E_{R}}=\left\{\left\|\nabla u_{0}\right\|_{L^{2}\left(B_{R}\right)}^{2}+\left\|u_{1}\right\|_{L^{2}\left(B_{R}\right)}^{2}\right\}^{1 / 2} .
$$

Hence it follows from (7.1) and (7.2) that

$$
\sqrt{E_{l o c, R}(t)} \leq C(R)\|u(0)\|_{E} \mathrm{e}^{-\lambda t}+\int_{0}^{t}\left\|U_{0}(t-\tau) \boldsymbol{F}\left(u_{t}(\tau)\right)\right\|_{E_{R}} d \tau,
$$

where we set

$$
E_{l o c, R}=\frac{1}{2} \int_{B_{R}}\left\{\left|u_{t}(t, x)\right|^{2}+|\nabla u(t, x)|^{2}\right\} d x
$$

If we set

$$
v(t, x ; \tau)=-\frac{t-\tau}{4 \pi} \int_{S^{2}}\left(a u_{t}^{2 m+1}\right)(\tau, x+(t-\tau) \omega) d \omega,
$$

then we see from the well-known Kirchhoff formula of the free wave equation in $\mathbb{R}^{3}$ that

$$
U_{0}(t-\tau) \boldsymbol{F}\left(u_{t}(\tau)\right)=\left\{v(t, x ; \tau), v_{t}(t, x ; \tau)\right\},
$$

which implies

$$
\begin{aligned}
& \| U_{0}(t-\tau) \boldsymbol{F}\left(u_{t}(\tau) \|_{E_{R}}^{2}\right. \\
= & \|\nabla v(t, x ; \tau)\|_{L^{2}\left(B_{R}\right)}^{2}+\left\|v_{t}(t, x ; \tau)\right\|_{L^{2}\left(B_{R}\right)}^{2} \\
\leq & C(1+t-\tau)^{2} \sum_{|\alpha| \leq 1} \int_{S^{2}}\left\|D_{x}^{\alpha}\left(a u_{t}^{2 m+1}\right)(\tau, x+(t-\tau) \omega)\right\|_{L^{2}\left(B_{R}\right)}^{2} d \omega \\
\leq & C(1+t-\tau)^{2} \sum_{|\alpha| \leq 1}\left\|D_{x}^{\alpha}\left(a u_{t}^{2 m+1}\right)\left(\tau, x+(t-\tau) \omega_{*}\right)\right\|_{L^{2}\left(B_{R}\right)}^{2}
\end{aligned}
$$

for some $C>0$ and $\omega_{*} \in S^{2}$, where we have used the identities

$$
\begin{aligned}
\nabla v(t, x ; \tau)= & -\frac{t-\tau}{4 \pi} \int_{S^{2}} \nabla\left(a u_{t}^{2 m+1}\right)(\tau, x+(t-\tau) \omega) d \omega \\
v_{t}(t, x ; \tau)= & -\frac{1}{4 \pi} \int_{S^{2}}\left(a u_{t}^{2 m+1}\right)(\tau, x+(t-\tau) \omega) d \omega \\
& -\frac{t-\tau}{4 \pi} \int_{S^{2}} \omega \cdot \nabla\left(a u_{t}^{2 m+1}\right)(\tau, x+(t-\tau) \omega) d \omega .
\end{aligned}
$$

Here we see from $|\nabla a| \leq C a$, the estimate $\left\|\nabla u_{t}(t)\right\|_{L^{\infty}}<\varepsilon_{0}$ and the decay estimate (2.11) that

$$
\begin{aligned}
& \sum_{|\alpha| \leq 1}\left\|D_{x}^{\alpha}\left(a u_{t}^{2 m+1}\right)\left(\tau, x+(t-\tau) \omega_{*}\right)\right\|_{L^{2}\left(B_{R}\right)}^{2} \\
\leq & C(1+\tau)^{-2 \eta}\left\{(1+\tau)^{-4 m-2}+\varepsilon_{0}^{2}(1+\tau)^{-4 m}\right\}\left\|\left\{1+\left|x+(t-\tau) \omega_{*}\right|\right\}^{-\delta}\right\|_{L^{2}\left(B_{R}\right)}^{2} \\
\leq & C\left(\varepsilon_{0}\right)(1+\tau)^{-4 m-2 \eta}\left\|\{1+|r-(t-\tau)|\}^{-\delta}\right\|_{L^{2}\left(B_{R}\right)}^{2}
\end{aligned}
$$


which implies

$$
\begin{aligned}
& \int_{0}^{t}\left\|U_{0}(t-\tau) \boldsymbol{F}\left(u_{t}\right)\right\|_{E_{R}} d \tau \\
\leq & C\left(\varepsilon_{0}\right) \int_{0}^{t}(1+\tau)(1+t-\tau)^{-2 m-\eta}\left\|(1+|r-\tau|)^{-\delta}\right\|_{L^{2}\left(B_{R}\right)} d \tau .
\end{aligned}
$$

Let $T_{0}$ be any fixed number with $T_{0}>R$. Then we have

$$
\begin{aligned}
& \int_{T_{0}}^{t}(1+\tau)(1+t-\tau)^{-2 m-\eta}\left\|(1+|r-\tau|)^{-\delta}\right\|_{L^{2}\left(B_{R}\right)} d \tau \\
\leq & C(R) \int_{T_{0}}^{t}(1+t-\tau)^{-2 m-\eta}(1+\tau)^{-(\delta-1)} d \tau .
\end{aligned}
$$

When $1<\delta \leq 2$, we see that

$$
\begin{aligned}
& \int_{T_{0}}^{t / 2}(1+t-\tau)^{-2 m-\eta}(1+\tau)^{-(\delta-1)} d \tau \\
\leq & C\left(T_{0}\right)\left(1+\frac{t}{2}\right)^{-2 m-\eta} \int_{T_{0}}^{t / 2}(1+\tau)^{-(\delta-1)} d \tau \\
\leq & \begin{cases}\frac{C\left(T_{0}\right)}{2-\delta}\left(1+\frac{t}{2}\right)^{-2 m-\eta-\delta+2}, & (1<\delta<2), \\
C\left(T_{0}\right)\left(1+\frac{t}{2}\right)^{-2 m-\eta} \log \left(1+\frac{t}{2}\right), & (\delta=2)\end{cases}
\end{aligned}
$$

and

$$
\begin{aligned}
& \int_{t / 2}^{t}(1+t-\tau)^{-2 m-\eta}(1+\tau)^{-(\delta-1)} d \tau \\
\leq & \left(1+\frac{t}{2}\right)^{-(\delta-1)} \int_{t / 2}^{t}(1+t-\tau)^{-2 m-\eta} d \tau \\
\leq & C\left(1+\frac{t}{2}\right)^{-(\delta-1)}
\end{aligned}
$$

for all $t>2 T_{0}$. Hence, it follows from (7.3) (7.8) that

$$
E_{l o c, R}(t) \leq C\left(R, \varepsilon_{0}\right)(1+t)^{-2(\delta-1)}
$$

for all $t>2 T_{0}$.

When $\delta>2$, we see that

$$
\begin{aligned}
& \int_{T_{0}}^{t / 2}(1+t-\tau)^{-2 m-\eta}(1+\tau)^{-(\delta-1)} d \tau \\
\leq & \left(1+\frac{t}{2}\right)^{-2 m-\eta} \int_{T_{0}}^{t / 2}(1+\tau)^{-(\delta-1)} d \tau \\
\leq & \frac{C\left(T_{0}\right)}{\delta-2}\left(1+\frac{t}{2}\right)^{-2 m-\eta}
\end{aligned}
$$


and

$$
\begin{aligned}
& \int_{t / 2}^{t}(1+t-\tau)^{-2 m-\eta}(1+\tau)^{-(\delta-1)} d \tau \\
\leq & \left(1+\frac{t}{2}\right)^{-(\delta-1)} \int_{t / 2}^{t}(1+t-\tau)^{-2 m-\eta} d \tau \\
\leq & \left(1+\frac{t}{2}\right)^{-(\delta-1)}
\end{aligned}
$$

for all $t>2 T_{0}$. Hence, it follows from (7.3), (7.5), (7.9) and (7.10) that

$$
E_{l o c, R}(t) \leq C\left(R, \varepsilon_{0}\right)(1+t)^{-\min \{2(2 m+\eta), 2(\delta-1)\}}
$$

for all $t>2 T_{0}$.

In any case we have

$$
E_{l o c, R}(t) \leq C\left(R, \varepsilon_{0}\right)(1+t)^{-\min \{2(2 m+\eta), 2(\delta-1)\}}
$$

for all $t>2 T_{0}$. Since $E_{l o c, R}(t)$ is bounded for $0 \leq t \leq 2 T_{0}$, we have the decay estimate (2.13), which completes the proof of Theorem 2.4.

\section{Appendix A. Proof of Proposition 5.1}

For $K>0$ and $T>0$, we define the space $X(T)$ as the set of all $v$ belonging to $C\left([0, T] ; H_{\nabla}(\Omega)\right)$ such that

$$
\begin{aligned}
& \nabla v \in C\left([0, T] ; H^{3}(\Omega)\right), \\
& v_{t} \in \bigcap_{j=0}^{2} C^{j}\left([0, T] ; H^{3-j}(\Omega) \cap H_{0}^{1}(\Omega)\right) \cap C^{3}\left([0, T] ; L^{2}(\Omega)\right), \\
& v(0, x)=u_{0}(x), \quad v_{t}(0, x)=u_{1}(x) \quad \text { in } \Omega, \\
& \|v\|_{X(T)} \leq K,
\end{aligned}
$$

where we set

$$
\begin{aligned}
& \|v\|_{X(T)} \\
= & \sup _{0 \leq t \leq T}\left\{\|\nabla v(t)\|_{H^{3}}+\sum_{j=0}^{3}\left\|D_{t}^{j+1} v(t)\right\|_{H^{3-j}}+\sum_{j=1}^{2}\left\|D_{t}^{j} v(t)\right\|_{L^{\infty}}+\|\nabla v(t)\|_{L^{\infty}}\right\} .
\end{aligned}
$$

Now we consider the initial-boundary value problem

$$
(\mathrm{P})_{v} \begin{cases}u_{t t}-\Delta u+a(t, x) v_{t}^{2 m+1}=0, & (t, x) \in(0, \infty) \times \Omega, \\ u(0, x)=u_{0}(x), \quad u_{t}(0, x)=u_{1}(x), & x \in \Omega, \\ u(t, x)=0, & (t, x) \in(0, \infty) \times \partial \Omega .\end{cases}
$$

Then the general theory of hyperbolic equations (cf. Mizohata [, p. 368, Theorem 6.12]) implies that if $v \in X(T)$, then the problem $(\mathrm{P})_{v}$ has a unique solution $u \in X(T)$. We define the map $\Phi$ as

$$
\Phi: v \longmapsto u .
$$

Proposition A.1. For $K>0$, if we choose $T_{0}>0$ and the size of the initial data so small that

$$
\left(C_{1}+C_{3}+C_{5}\right)\left\{\left\|\nabla u_{0}\right\|_{H^{3}}+\sum_{j=0}^{3}\left\|D_{t}^{j+1} u(0)\right\|_{H^{3-j}}\right\}<\frac{K}{2},
$$




$$
\left(C_{2}+C_{4}+C_{6}\right) K^{2 m} T_{0}<\frac{1}{2},
$$

then $\Phi$ maps $X\left(T_{0}\right)$ into itself. Here, the constants $C_{j}(j=1,2, \ldots, 6)$ are determined in the course of the proof.

Proof. First we shall prove that

$$
\sum_{j=0}^{l} \sqrt{E\left(D_{t}^{j} u(t)\right)} \leq \sum_{j=0}^{l} \sqrt{E\left(D_{t}^{j} u(0)\right)}+C K^{2 m+1} T \quad(l=0,1,2,3)
$$

for $0 \leq t \leq T$.

For this, we set $\sigma_{l}\left(a, v_{t}\right)=D_{t}^{l}\left(a v_{t}^{2 m+1}\right)(l=0,1,2,3)$, that is,

$$
\begin{aligned}
\sigma_{0}\left(a, v_{t}\right)= & a v_{t}^{2 m+1} \\
\sigma_{1}\left(a, v_{t}\right)= & a_{t} v_{t}^{2 m+1}+(2 m+1) a v_{t}^{2 m} D_{t}^{2} v, \\
\sigma_{2}\left(a, v_{t}\right)= & \left(D_{t}^{2} a\right) v_{t}^{2 m+1}+(4 m+2) a_{t} v_{t}^{2 m} D_{t}^{2} v+m(4 m+2) a v_{t}^{2 m-1}\left(D_{t}^{2} v\right)^{2} \\
& +(2 m+1) a v_{t}^{2 m} D_{t}^{3} v, \\
\sigma_{3}\left(a, v_{t}\right)= & \left(D_{t}^{3} a\right) v_{t}^{2 m+1}+(6 m+3)\left(D_{t}^{2} a\right) v_{t}^{2 m} D_{t}^{2} v \\
& +(6 m+3) a_{t}\left\{2 m v_{t}^{2 m-1}\left(D_{t}^{2} v\right)^{2}+v_{t}^{2 m} D_{t}^{3} v\right\} \\
& +2 m(2 m+1) a\left\{(2 m-1) v_{t}^{2 m-2}\left(D_{t}^{2} v\right)^{3}+3 v_{t}^{2 m-1}\left(D_{t}^{2} v\right)\left(D_{t}^{3} v\right)\right\} \\
& +(2 m+1) a v_{t}^{2 m} D_{t}^{4} v .
\end{aligned}
$$

Differentiating the equation in $(\mathrm{P})_{v} l$ times with respect to $t(l=0,1,2,3)$ and multiplying it by $D_{t}^{l+1} u(t)$, we have

$$
E\left(D_{t}^{l} u(t)\right) \leq E\left(D_{t}^{l} u(0)\right)+\sqrt{2} \int_{0}^{t}\left\|\sigma_{l}\left(a, v_{t}\right)\right\| \sqrt{E\left(D_{t}^{l} u(\tau)\right)} d \tau,
$$

which implies

$$
\sqrt{E\left(D_{t}^{l} u(t)\right)} \leq \sqrt{E\left(D_{t}^{l} u(0)\right)}+\sqrt{2} \int_{0}^{t}\left\|\sigma_{l}\left(a, v_{t}\right)\right\| d \tau
$$

for $0 \leq t \leq T$. If we can show that

$$
\left\|\sigma_{l}\left(a, v_{t}\right)\right\| \leq C K^{2 m} \sum_{j=1}^{l+1}\left\|D_{t}^{j} v\right\|
$$

for some $C>0$, it will follow from (A.4) and $v \in X(T)$ that (A.3) is valid.

Now we estimate $\left\|\sigma_{l}\left(a, v_{t}\right)\right\|$ in the four cases $l=0,1,2,3$, respectively.

Case $l=0$ :

$$
\left\|\sigma_{0}\left(a, v_{t}\right)\right\| \leq\left\|a v_{t}^{2 m+1}\right\| \leq a_{1} K^{2 m}\left\|v_{t}\right\| .
$$

Case $l=1$ :

$$
\begin{aligned}
\left\|\sigma_{1}\left(a, v_{t}\right)\right\| & \leq\left\|a_{t} v_{t}^{2 m+1}\right\|+(2 m+1)\left\|a v_{t}^{2 m} D_{t}^{2} v\right\| \\
& \leq C K^{2 m}\left(\left\|v_{t}\right\|+\left\|D_{t}^{2} v\right\|\right) .
\end{aligned}
$$

Case $l=2$ :

$$
\begin{aligned}
\left\|\sigma_{2}\left(a, v_{t}\right)\right\| & \leq C\left\{\left\|v_{t}^{2 m+1}\right\|+\left\|v_{t}^{2 m} D_{t}^{2} v\right\|+\left\|v_{t}^{2 m-1}\left(D_{t}^{2} v\right)^{2}\right\|+\left\|v_{t}^{2 m} D_{t}^{3} v\right\|\right\} \\
& \leq C K^{2 m}\left(\left\|v_{t}\right\|+\left\|D_{t}^{2} v\right\|+\left\|D_{t}^{3} v\right\|\right) .
\end{aligned}
$$


Case $l=3$ :

$$
\begin{aligned}
\left\|\sigma_{2}\left(a, v_{t}\right)\right\| \leq & C\left\{\sum_{j=0}^{2}\left\|v_{t}^{2 m+1-j}\left(D_{t}^{2} v\right)^{j}\right\|+\left\|v_{t}^{2 m-2}\left(D_{t}^{2} v\right)^{3}\right\|+\left\|v_{t}^{2 m} D_{t}^{3} v\right\|\right. \\
& \left.+\left\|v_{t}^{2 m-1}\left(D_{t}^{2} v\right)\left(D_{t}^{3} v\right)\right\|+\left\|v_{t}^{2 m} D_{t}^{4} v\right\|\right\} \\
\leq & C K^{2 m}\left(\left\|v_{t}\right\|+\left\|D_{t}^{2} v\right\|+\left\|D_{t}^{3} v\right\|+\left\|D_{t}^{4} v\right\|\right) .
\end{aligned}
$$

Thus we have A.3.

Next we shall prove that

$$
\sum_{j=0}^{3}\left\|D_{t}^{j+1} u(t)\right\|_{H^{3-j}} \leq C_{1} \sum_{j=0}^{3}\left\|D_{t}^{j+1} u(0)\right\|_{H^{3-j}}+C_{2} K^{2 m+1} T
$$

for some $C_{j}>0(j=1,2)$ and $0 \leq t \leq T$. In fact, recalling the definition of $\sigma_{\Delta}$ (see (5.39) $)$, we see by the same calculations as in Proposition 5.6 that

$$
\begin{aligned}
\sqrt{E(\Delta u(t))} & \leq \sqrt{E(\Delta u(0))}+C K^{2 m} \int_{0}^{t}\left\{\left\|\Delta v_{t}(\tau)\right\|+\left\|\sigma_{\Delta}\left(a, v_{t}\right)\right\|\right\} d \tau \\
& \leq \sqrt{E(\Delta u(0))}+C K^{2 m} \int_{0}^{t}\left\{\left\|\Delta v_{t}(\tau)\right\|+\left\|v_{t}(\tau)\right\|+\left\|\nabla v_{t}(\tau)\right\|\right\} d \tau .
\end{aligned}
$$

Since $v \in X(T)$, we have

$$
\left\|\Delta v_{t}(\tau)\right\|+\left\|v_{t}(\tau)\right\|+\left\|\nabla v_{t}(\tau)\right\| \leq 3 K
$$

which implies that

$$
\sqrt{E(\Delta u(t))} \leq \sqrt{E(\Delta u(0))}+C K^{2 m+1} T .
$$

Differentiating the equation in $(\mathrm{P})_{v}$ with respect to $t$, and then differentiating the result twice with respect to $x_{j}$, we have

$$
\left(\Delta u_{t}\right)_{t t}-\Delta\left(\Delta u_{t}\right)+D_{t}\left(\Delta\left(a v_{t}^{2 m+1}\right)\right)=0 .
$$

Multiplying this by $\Delta u_{t t}$, we have

$$
\begin{aligned}
& E\left(\Delta u_{t}(t)\right) \\
\leq & E\left(\Delta u_{t}(0)\right)+\int_{0}^{t}\left\{(2 m+1)\left\|D_{t}\left(a v_{t}^{2 m} \Delta v_{t}\right)\right\|+\left\|D_{t} \sigma_{\Delta}\left(a, v_{t}\right)\right\|\right\} \sqrt{E\left(\Delta u_{t}(\tau)\right)} d \tau,
\end{aligned}
$$

which implies

$$
\sqrt{E\left(\Delta u_{t}(t)\right)} \leq \sqrt{E\left(\Delta u_{t}(0)\right)}+\int_{0}^{t}\left\{(2 m+1)\left\|D_{t}\left(a v_{t}^{2 m} \Delta v_{t}\right)\right\|+\left\|D_{t} \sigma_{\Delta}\left(a, v_{t}\right)\right\|\right\} d \tau .
$$

Here,

$$
\begin{aligned}
& (2 m+1)\left\|D_{t}\left(a v_{t}^{2 m} \Delta v_{t}\right)\right\|+\left\|D_{t} \sigma_{\Delta}\left(a, v_{t}\right)\right\| \\
\leq & C K^{2 m}\left(\left\|v_{t}\right\|+\left\|D_{t}^{2} v\right\|+\left\|\nabla v_{t}\right\|+\left\|\nabla v_{t t}\right\|+\left\|\Delta v_{t}\right\|+\left\|\Delta v_{t t}\right\|\right) .
\end{aligned}
$$

Hence it follows from (A.8) and (A.9) that

$$
\sqrt{E\left(\Delta u_{t}(t)\right)} \leq \sqrt{E\left(\Delta u_{t}(0)\right)}+C_{2} K^{2 m+1} T \text {. }
$$


Therefore, it follows from Lemma 3.1, (A.3), A.7) and (A.10) that

$$
\begin{aligned}
&\left\|u_{t}(t)\right\|_{H^{3}} \leq C\left\{\left\|\Delta u_{t}(t)\right\|_{H^{1}}+\left\|u_{t}(t)\right\|\right\} \\
& \leq C\left\{\sum_{j=0}^{1} \sqrt{E\left(\Delta D_{t}^{j} u(0)\right)}+\sqrt{E(u(0))}+C_{2} K^{2 m+1} T\right\}, \\
&\left\|D_{t}^{2} u(t)\right\|_{H^{2}} \leq\left\|\Delta D_{t}^{2} u(t)\right\|+\left\|D_{t}^{2} u(t)\right\| \\
& \leq C\left\{\sqrt{E\left(\Delta u_{t}(0)\right)}+\sqrt{E\left(D_{t}^{2} u(0)\right)}+K^{2 m+1} T\right\}
\end{aligned}
$$

and

$$
\left\|D_{t}^{3} u(t)\right\|_{H^{1}}+\left\|D_{t}^{4} u(t)\right\| \leq C\left\{\sum_{j=1}^{3} \sqrt{E\left(D_{t}^{j} u(0)\right)}+K^{2 m+1} T\right\} .
$$

From (A.11), (A.12) and (A.13), we obtain (A.6).

Finally, we shall show that

$$
\|\nabla u(t)\|_{H^{3}} \leq C_{3}\left\{\left\|\nabla u_{0}\right\|_{H^{3}}+\sum_{j=0}^{3}\left\|D_{t}^{j+1} u(0)\right\|_{H^{3-j}}\right\}+C_{4} K^{2 m+1} T
$$

and

$$
\begin{aligned}
& \sum_{j=0}^{1}\left\|D_{t}^{j} u_{t}(t)\right\|_{L^{\infty}}+\left\|\nabla u_{t}(t)\right\|_{L^{\infty}} \\
\leq & C_{5}\left\{\left\|\nabla u_{0}\right\|_{H^{3}}+\sum_{j=0}^{3}\left\|D_{t}^{j+1} u(0)\right\|_{H^{3-j}}\right\}+C_{6} K^{2 m+1} T
\end{aligned}
$$

for $0 \leq t \leq T$.

Multiplying the equation in $(\mathrm{P})_{v}$ by $-\Delta u_{t}$, we easily see that

$$
\|\Delta u(t)\| \leq C\left\{\|\Delta u(0)\|+\sqrt{E\left(u_{t}(0)\right)}+K^{2 m+1} T\right\} .
$$

It follows from Lemma 3.2 and A.16 that

$$
\begin{aligned}
\|\nabla u(t)\|_{H^{1}} & \leq \sum_{|\alpha|=2}\left\|D_{x}^{\alpha} u(t)\right\|+\|\nabla u(t)\| \\
& \leq C\{\|\Delta u(t)\|+\|\nabla u(t)\|\} \\
& \leq C\left\{\sqrt{E(u(0))}+\sqrt{E\left(u_{t}(0)\right)}+\|\Delta u(0)\|+K^{2 m+1} T\right\} .
\end{aligned}
$$

Combining (A.17) with Lemma 3.2, (A.7) and (A.16), we see that

$$
\begin{aligned}
\|\nabla u(t)\|_{H^{2}} & \leq\|\nabla u(t)\|_{H^{1}}+\sum_{|\alpha|=2}\left\|D_{x}^{\alpha} \nabla u(t)\right\| \\
& \leq C\left\{\|\nabla u(t)\|_{H^{1}}+\|\nabla \Delta u(t)\|\right\} \\
& \leq C\left\{\sqrt{E(u(0))}+\sqrt{E(\Delta u(0))}+\left\|\Delta u_{0}\right\|+K^{2 m+1} T\right\} .
\end{aligned}
$$


Further, differentiating the equation in $(\mathrm{P})_{v}$ twice with respect to $x_{j}$ and multiplying the result by $-\Delta\left(\Delta u_{t}\right)$, we see that

$$
\begin{aligned}
& \frac{1}{2} \frac{d}{d t}\left\{\left\|\nabla \Delta u_{t}(t)\right\|^{2}+\|\Delta(\Delta u(t))\|^{2}\right\} \\
\leq & \int_{\Omega}\left\{(2 m+1)\left|\nabla\left(a v_{t}^{2 m} \Delta v_{t}\right)\right|+\left|\nabla \sigma_{\Delta}\left(a, v_{t}\right)\right|\right\}\left|\nabla \Delta u_{t}(t)\right| d x,
\end{aligned}
$$

which implies

$$
\begin{aligned}
& \left\|\nabla \Delta u_{t}(t)\right\|+\|\Delta(\Delta u(t))\| \\
\leq & \left\|\nabla \Delta u_{t}(0)\right\|+\|\Delta(\Delta u(0))\|+C \int_{0}^{t}\left\{\left\|\nabla\left(a v_{t}^{2 m} \Delta v_{t}\right)\right\|+\left\|\nabla \sigma_{\Delta}\left(a, v_{t}\right)\right\|\right\} d \tau \\
\leq & \left\|\nabla \Delta u_{t}(0)\right\|+\|\Delta(\Delta u(0))\|+C K^{2 m} \int_{0}^{t}\left\|v_{t}(\tau)\right\|_{H^{3}} d \tau \\
\leq & C\left\{\left\|\nabla \Delta u_{t}(0)\right\|+\|\Delta(\Delta u(0))\|+K^{2 m+1} T\right\} .
\end{aligned}
$$

By the same argument as in Proposition 5.7 we have

$$
\begin{aligned}
\|\nabla u(t)\|_{H^{3}} & \leq C\left\{\|\nabla u(t)\|_{H^{2}}+\sum_{|\alpha|=2}\left\|D_{x}^{\alpha} \Delta u(t)\right\|+\sum_{|\alpha|=2}\left\|D_{x}^{\alpha} \nabla u(t)\right\|\right\} \\
& \leq C\left\{\|\nabla u(t)\|_{H^{2}}+\sum_{|\alpha|=2}\left\|D_{x}^{\alpha} \Delta u(t)\right\|+\|\nabla \Delta u(t)\|\right\} .
\end{aligned}
$$

Here, we see from Lemma 3.2, A.7 and that

$$
\begin{aligned}
\sum_{|\alpha|=2}\left\|D_{x}^{\alpha} \Delta u(t)\right\| & \leq C\{\|\Delta(\Delta u(t))\|+\|\nabla \Delta u(t)\|\} \\
& \leq C\left\{\left\|\nabla \Delta u_{t}(0)\right\|+\|\Delta(\Delta u(0))\|+\sqrt{E(\Delta u(0))}+K^{2 m+1} T\right\} .
\end{aligned}
$$

Thus, combining this with (A.16), (A.18), A.19) and (A.20), we obtain A.14). Further, it follows from Sobolev's inequality that

$$
\sum_{j=0}^{1}\left\|D_{t}^{j} u_{t}(t)\right\|_{L^{\infty}}+\left\|\nabla u_{t}(t)\right\|_{L^{\infty}} \leq C\left\{\left\|u_{t}(t)\right\|_{H^{3}}+\left\|u_{t t}(t)\right\|_{H^{2}}\right\} .
$$

Combining this with A.6. we have A.15).

If we take a number $T_{0}>0$ and the size of the initial data $\left\{u_{0}, u_{1}\right\}$ as in (A.1) and (A.2), then we conclude from (A.6), (A.14) and (A.15) that $u \in X\left(T_{0}\right)$, which implies $\Phi$ maps $X\left(T_{0}\right)$ into itself.

We construct a local solution to the problem $(\mathrm{P})_{m}$. Let $u^{0}$ be any element in $X\left(T_{0}\right)$. We define $\left\{u^{j}\right\}_{j=0}^{\infty}$ by

$$
u^{j+1}=\Phi u^{j}, \quad j=0,1,2, \cdots .
$$

In other words, $\left\{u^{j}\right\}$ is recursively determined by

$$
(\mathrm{P})_{j} \begin{cases}u_{t t}^{j}-\Delta u^{j}+a(t, x)\left(D_{t} u^{j-1}\right)^{2 m+1}=0, & (t, x) \in\left(0, T_{0}\right) \times \Omega, \\ u^{j}(0, x)=u_{0}(x), \quad u_{t}^{j}(0, x)=u_{1}(x), & x \in \Omega, \\ u^{j}(t, x)=0, & (t, x) \in\left(0, T_{0}\right) \times \partial \Omega .\end{cases}
$$


By Proposition A.1 we see that $u^{j+1} \in X\left(T_{0}\right)$. If we set $w^{j}=u^{j}-u^{j-1}$, then $w^{j}$ satisfies the following initial-boundary value problem in $\left(0, T_{0}\right) \times \Omega$ :

$$
(\mathrm{P})_{j}^{\prime} \begin{cases}\square w^{j}+a(t, x)\left\{\left(D_{t} u^{j-1}\right)^{2 m+1}-\left(D_{t} u^{j-2}\right)^{2 m+1}\right\}=0, & (t, x) \in\left(0, T_{0}\right) \times \Omega, \\ w^{j}(0, x)=w_{t}^{j}(0, x)=0, & x \in \Omega, \\ w^{j}(t, x)=0, & (t, x) \in\left(0, T_{0}\right) \times \partial \Omega,\end{cases}
$$

where $\square=D_{t}^{2}-\Delta$. Thus, in a way completely analogous to the proof of Proposition A.1 we conclude that $\left\|w^{j}\right\|_{X\left(T_{0}\right)}$ converges to 0 as $j \rightarrow \infty$. Hence $\left\{u^{j}\right\}_{j=0}^{\infty}$ is a Cauchy sequence in $X\left(T_{0}\right)$, and the limit function $u$, which is a weak solution in time, satisfies the original problem $(\mathrm{P})_{m}$. Therefore, the same argument as in the proof of Theorem 2.2 (i) implies that this $u$ satisfies the original problem $(\mathrm{P})_{m}$ in the strong sense. The uniqueness part is standard. So we shall omit it. This completes the proof.

\section{REFERENCES}

[1] D. Gilbarg and N. S. Trudinger, Elliptic partial differential equations of second order, 2nd ed., Springer-Verlag, 1983. MR 86c:35035

[2] N. Hayashi, Global existence of small solutions to quadratic nonlinear wave equations in an exterior domain, J. Funct. Anal. 131 (1995), 302-344. MR 96f:35113

[3] M. Ikawa, Hyperbolic partial differential equations and wave phenomena, Transl. Math. Monogr., Vol. 189, Amer. Math. Soc., 2000. MR 2001j:35176

[4] O. A. Ladyzhenskaya, The mathematical theory of viscous incompressible flow, Revised 2nd ed., New York: Gordon and Breach, 1969. MR 40:7610

[5] J. L. Lions and W. A. Strauss, Some nonlinear evolution equations, Bull. Soc. Math. France 93 (1965), 43-96. MR 33:7663

[6] T. Matsuyama, Asymptotic behaviour of solutions to the initial-boundary value problem with an effective dissipation around the boundary, J. Math. Anal. Appl. 271 (2002), 467-492.

[7] T. Matsuyama, Asymptotic behaviour of solutions for the nonlinear dissipative wave equations, preprint (2001).

[8] S. Mizohata, The theory of partial differential equations, Cambridge Univ. Press, 1973. MR 58:29033

[9] K. Mochizuki, Decay and asymptotics for wave equations with dissipative term, Lecture Notes in Phys. 39, 1975, Springer-Verlag, pp. 486-490. MR 58:29089

[10] K. Mochizuki, Scattering theory for wave equations (in Japanese), Kinokuniya, 1984.

[11] K. Mochizuki and T. Motai, On energy decay-nondecay problems for the wave equations with nonlinear dissipative term in $\mathbb{R}^{N}$, J. Math. Soc. Japan 47 (1995), 405-421. MR 96c:35122

[12] K. Mochizuki and H. Nakazawa, Energy decay and asymptotic behavior of solutions to the wave equations with linear dissipation, Publ. RIMS, Kyoto Univ. 32 (1996), 401-414. MR 97g:35101

[13] C. Morawetz, Exponential decay of solutions of the wave equations, Comm. Pure Appl. Math. 19 (1966), 439-444. MR 34:4664

[14] T. Motai and K. Mochizuki, On asymptotic behaviors for wave equations with a nonlinear dissipative term in $\mathbb{R}^{N}$, Hokkaido Math. J. 25 (1996), 119-135. MR 96m:35226

[15] M. Nakao, Existence of global classical solutions of the initial-boundary value problem for some nonlinear wave equations, J. Math. Anal. Appl. 146 (1990), 217-240. MR 91d:35141

[16] M. Nakao, Stabilization of local energy in an exterior domain for the wave equation with a localized dissipation, J. Differential Equations 148 (1998), 388-406. MR 2000c:35141

[17] J. Sather, The existence of a global classical solution of the initial-boundary value problem for $\square u+u^{3}=f$, Arch. Rational Mech. Anal. 22 (1966), 129-135. MR 33:6124

[18] J. Shatah, Global existence of small solutions to nonlinear evolution equations, J. Differential Equations 46 (1982), 409-425. MR 84g:35036

[19] Y. Shibata and Y. Tsutsumi, Global existence theorem of nonlinear wave equations in the exterior domain, Lecture Notes in Num. Appl. Anal. 6 (1983), 155-196, Kinokuniya/NorthHolland. MR 87f:35161 
[20] Y. Shibata and Y. Tsutsumi, On a global existence theorem of small amplitude solutions for nonlinear wave equations in an exterior domain, Math. Z. 191 (1986), 165-199. MR 87i:35122

Department of Mathematics, Tokai University, Hiratsuka, Kanagawa 259-1292, Japan

E-mail address: matsu@sm.u-tokai.ac.jp 